\title{
3 Research Square \\ RUFY3 and RUFY4 are ARL8 effectors that couple lysosomes to dynein-dynactin
}

\section{Tal Keren-Kaplan}

Eunice Kennedy Shriver National Institute of Child Health and Human Development, National Institutes of Health

Amra Saric

$\mathrm{NIH} / \mathrm{NICHD}$

\section{Saikat Ghosh}

Eunice Kennedy Shriver National Institute of Child Health and Human Development, National Institutes of Health

\section{Chad Williamson}

$\mathrm{NIH}$

\section{Rui Jia}

Eunice Kennedy Shriver National Institute of Child Health and Human Development, National Institutes of Health

Yan Li

NINDS https://orcid.org/0000-0003-3820-5960

Juan Bonifacino ( $\sim$ bonifacinoj@mail.nih.gov )

National Institutes of Health

\section{Article}

Keywords: ARL8, RUFY3, RUFY4, lysosome transport

Posted Date: May 25th, 2021

DOl: https://doi.org/10.21203/rs.3.rs-469512/v1

License: (c) (i) This work is licensed under a Creative Commons Attribution 4.0 International License. Read Full License

Version of Record: A version of this preprint was published at Nature Communications on March 21st, 2022. See the published version at https://doi.org/10.1038/s41467-022-28952-y. 


\section{RUFY3 and RUFY4 are ARL8 effectors that couple lysosomes to dynein-dynactin}

Tal Keren-Kaplan ${ }^{1}$, Amra Saric ${ }^{1}$, Saikat Ghosh ${ }^{1}$, Chad Williamson ${ }^{1}$, Rui Jia ${ }^{1}$, Yan Li ${ }^{2}$ and Juan S. Bonifacino ${ }^{1^{*}}$

$13{ }^{2}$ Proteomics Core Facility, National Institute of Neurological Disorders and Stroke, National Institutes of Health, Bethesda, Maryland, USA.

15 
22 The small GTPase ARL8 associates with lysosomes and recruits several effectors that mediate

23 coupling to kinesins for anterograde transport, as well as tethering for eventual fusion with

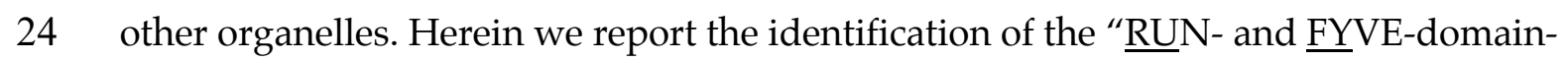

25 containing" proteins RUFY3 and RUFY4 as novel ARL8 effectors that couple lysosomes to

26 dynein-dynactin for retrograde transport. Using various biochemical approaches, we find that

27 RUFY3/4 interact with both GTP-bound ARL8 and dynein-dynactin. In addition, we show that

28 RUFY3/4 are both necessary and sufficient for concentration of lysosomes in the juxtanuclear

29 area of the cell. RUFY3/4 also promote retrograde transport of lysosomes in the axon of

30 hippocampal neurons. The function of RUFY3/4 in retrograde transport is required for

31 juxtanuclear redistribution of lysosomes upon serum starvation or cytoplasmic alkalinization,

32 and may underlie the reported roles of RUFY3/4 in axon development/degeneration, cancer

33 and immunity. These studies thus establish RUFY3/4 as novel ARL8-dependent, dynein-

34 dynactin adaptors, and highlight the role of ARL8 in the regulation of both anterograde and

35 retrograde lysosome transport. 


\section{Introduction}

The ADP-ribosylation factor (ARF) family of small GTPases comprises $~ 30$ members that regulate various aspects of cell physiology (Sztul et al., 2019). Among these members, the mammalian ARL8A and ARL8B paralogs (referred to indistinctly as "ARL8" unles otherwise specified) are unique in their ability to associate with lysosomes and to regulate multiple lysosomal functions (Khatter et al., 2015b) (for simplicity, herein we use the term "lysosomes" to denote lysosomes, late endosomes and related endosolysosomal organelles). Like other small GTPases, ARL8 cycles between GDP-bound, inactive and GTP-bound, active forms (Khatter et al., 2015b; Sztul et al., 2019). Whereas the GDP-bound form is cytosolic, the GTP-bound form associates with lysosomes (Bagshaw et al., 2006; Hofmann and Munro, 2006). The association of ARL8 with lysosomes depends on an N-terminal acetylated, amphipathic $\alpha$-helix (Hofmann and Munro, 2006), which, by analogy with other members of the ARF family (Antonny et al., 1997; Liu et al., 2009), likely swings out from the rest of the molecule upon GTP binding to mediate interaction with the lipid bilayer. In addition, this association requires the lysosome-associated hetero-octameric complex BORC (Pu et al., 2015), which may function as a guanine-nucleotideexchange factor (GEF) for the conversion of GDP-bound to GTP-bound ARL8 (Niwa et al., 2017).

The regulation of cellular functions by small GTPases is generally mediated by effectors that interact with the GTP-bound forms, resulting in recruitment and / or allosteric activation of the effectors (Sztul et al., 2019). Several effectors have been identified for mammalian ARL8, including the hetero-hexameric tethering complex HOPS (Garg et al., 2011), the adaptor proteins PLEKHM1 (Marwaha et al., 2017) and PLEKHM2 (also known as SKIP, the name used here) (Boucrot et al., 2005; Rosa-Ferreira and Munro, 2011), and the kinesin-3 motor protein KIF1 (Niwa et al., 2016). The interaction of ARL8-GTP with HOPS promotes fusion of lysosomes with late endosomes (Khatter et al., 2015a; Marwaha et al., 2017), phagosomes (Garg et al., 2011), autophagosomes (Jia et al., 2017) and Salmonella-containing vacuoles (Sindhwani et al., 2017), in some cases in cooperation with PLEKHM1 (Marwaha et al., 2017). The interaction of ARL8-GTP with SKIP mediates recruitment of the kinesin-1 motor protein $\left(\mathrm{KIF}_{2}-\mathrm{KLC}_{2}\right)$ for anterograde transport of lysosomes toward the peripheral cytoplasm in non-polarized cells (Guardia et al., 2016; Keren-Kaplan and Bonifacino, 2021; Pu et al., 2015; Rosa-Ferreira and Munro, 2011; Tuli et al., 2013) and the distal axon in neurons (Farias et al., 2017; Rosa-Ferreira et al., 2018; Vukoja et al., 2018). The ARL8-SKIP-kinesin-1 ensemble is also responsible for the formation of tubular lysosomes in lipopolysaccharide-treated macrophages (Mrakovic et al., 
71 2012) and in the process of phagolysosome resolution (Levin-Konigsberg et al., 2019). Finally,

72 while kinesin-1 requires SKIP for interaction with ARL8-GTP, kinesin-3 interacts directly with 73 ARL8-GTP (Niwa et al., 2016), also promoting anterograde transport of lysosomes toward the 74 cell periphery in non-polarized mammalian cells (Guardia et al., 2016), as well as lysosomes, 75 synaptic vesicle precursors, presynaptic active zone proteins, and dense core vesicles in $C$. 76 elegans and Drosophila neurons (Klassen et al., 2010; Lund et al., 2021; Niwa et al., 2016; Rosa-

77 Ferreira et al., 2018; Vukoja et al., 2018). Through these interactions, ARL8 regulates various 78 cellular processes mediated by lysosomes (Ballabio and Bonifacino, 2020), including endocytic 79 degradation (Khatter et al., 2015a; Oka et al., 2017), autophagy (Farias et al., 2017; Korolchuk et 80 al., 2011; Marwaha et al., 2017; Pu et al., 2015), microbial killing and antigen presentation (Garg 81 et al., 2011), natural killer cell cytotoxicity (Tuli et al., 2013), mTOR signaling (Jia and

82 Bonifacino, 2019; Korolchuk et al., 2011), cell adhesion and migration (Schiefermeier et al., 2014), 83 invasive cancer growth (Dykes et al., 2016), axonal growth-cone dynamics (Farias et al., 2017), 84 axon branching (Adnan et al., 2020), and egress of $\beta$-coronaviruses from infected cells (Ghosh et 85 al., 2020).

Although the number of known effectors and functions of ARL8 may seem large, there are ARFfamily GTPases that have many more. For example, ARF1 has more than 15 known effectors, each of which mediates a different function (Jackson and Bouvet, 2014; Sztul et al., 2019). It is thus possible that ARL8 has an even larger set of effectors and functions than are currently known. Herein we report the results of a search for additional ARL8 effectors using MitoID

92 (Gillingham et al., 2019), a method involving proximity biotinylation with mitochondrially-

93 targeted forms of human ARL8A and ARL8B, followed by isolation of biotinylated proteins and 94 mass spectrometry. Using this method, we identify the "ㄴNN and FYVE domain-containing"

95 proteins RUFY3 and RUFY4 (Char and Pierre, 2020) as novel ARL8 effectors. We find that ARL8 96 mediates recruitment of RUFY3/4 to lysosomes, promoting lysosome redistribution toward the 97 juxtanuclear area of the cell. Moreover, we show that RUFY3/4 increase retrograde transport of 98 lysosomes along the axon of rat hippocampal neurons. Further biochemical and cellular 99 analyses demonstrate that RUFY3 and RUFY4 also interact with the retrograde microtubule 100 motor dynein-dynactin, via interaction with the dynein light intermediate chain (LIC). These 101 findings identify RUFY3 and RUFY4 as novel ARL8-dependent adaptors that couple lysosomes 102 to dynein-dynactin for retrograde transport along microtubules. ARL8 can thus regulate both 103 anterograde and retrograde lysosome transport through interactions with different effectors 104 that link lysosomes to kinesin and dynein-dynactin motors, respectively. 
105

106

107

108

109

110

111

112

113

114

115

116

117

118

119

120

121

122

123

124

125

126

127

128

129

130

131

132

133

134

135

136

137

138

139

\section{Results}

\section{Identification of RUFY3 and RUFY4 as ARL8 effectors}

To identify ARL8 effectors, we used a modification of the MitoID procedure previously developed to identify interactors of RAB GTPases (Gillingham et al., 2019) (Supplementary Fig. 1a). We attached a mitochondrial-targeting sequence (MTS) from the outer mitochondrial membrane protein TOM20 (Kanaji et al., 2000), followed by the BioID2 biotin ligase (Kim et al.,

2016), to the GTP-bound (Q75L) or GDP-bound (T34N) forms of ARL8A and ARL8B lacking the N-terminal amphipathic $\alpha$-helix (Mito-ARL8 constructs) (Fig. 1a). MTS-BioID2 without ARL8 was used as a negative control (Fig. 1a). The Mito-ARL8 and control constructs were expressed by transient transfection into HEK293T cells, after which cells were incubated with $50 \mu \mathrm{M}$ biotin for $24 \mathrm{~h}$. Cells were then extracted with detergent, and biotinylated proteins were captured on neutravidin-agarose beads and identified by mass spectrometry (Supplementary Fig. 1a). Data were analyzed by comparing the abundance of proteins labeled by MTS-BioID2-ARL8-Q75L relative to the MTS-BioID2 control vs. Mito-BioID2-ARL8-T34N relative to a Mito-BioID2 control for both ARL8A (Fig. 1b) and ARL8B (Fig. 1c) (Supplementary Dataset 1). A top hit in these analyses was the known ARL8 effector PLEKHM2 (SKIP) (Rosa-Ferreira and Munro, 2011), which was only detected in isolates from the Q75L form of both ARL8A (Fig. 1b) and ARL8B (Fig. 1C). The identification of SKIP verified the reliability of the assay.

Interestingly, another top hit for both the ARL8A (Fig. 1b) and ARL8B (Fig. 1c) constructs was a protein named RUFY3 (also known as SINGAR or ZFYVE30) (Mori et al., 2007) (Fig. 1d). RUFY3 is one of 4 members of the RUFY family of proteins in humans (Char and Pierre, 2020). These proteins comprise, in N- to C-terminal direction, RUN, coiled-coil (CC) and FYVE domains joined by disordered sequences (Fig. 1d). RUFY3, in particular, has two CC domains (CC1 and CC2) and exists as 6 spliceforms, two of which are the 620-amino-acid RUFY3.1 (transcript variant 1) (NM_001037442) (also known as RUFY3XL, (Char and Pierre, 2020) and the 469amino-acid RUFY3.2 (transcript variant 2) (NM_014961) (Fig. 1d). Whereas RUFY3.1 includes all the domains of the RUFY family, RUFY3.2 lacks a C-terminal region comprising part of the CC2 domain and the entire FYVE domain (Fig. 1d). The shorter RUFY3.2 is the only RUFY3 spliceform characterized to date; previous studies showed that it plays roles in neuronal polarity and axon formation and degeneration (Hertz et al., 2019; Honda et al., 2017; Mori et al., 2007; Wei et al., 2014), and in cancer cell migration, invasion and metastasis (Men et al., 2019; Wang et al., 2015; Xie et al., 2017; Zhu et al., 2019). Protein and mRNA expression databases (e.g., https: / / www.proteinatlas.org/) indicate that RUFY3 is expressed in all cells and tissues, 
140 although with higher expression levels in the brain. Our mass spectrometric analyses identified

1416 peptides derived from the longer RUFY3.1 spliceform (Supplementary Fig. 1b), demonstrating 142 that this species is expressed in HEK293T cells.

144 To confirm the identification of RUFY3 as an ARL8 effector and to determine whether other 145 members of the RUFY family also interact with ARL8, we examined the intracellular 146 localization of GFP-tagged forms of the RUFY proteins co-expressed with MTS-BioID2-ARL8B147 T34N and Mito-BioID2-ARL8B-Q75L in HeLa cells (Fig. 1e,f). We observed that, in the presence 148 of MTS-BioID2-ARL8B-T34N, GFP-tagged RUFY1, RUFY2, RUFY3.1 and RUFY4 localized to the 149 cytosol and to a cluster in the juxtanuclear area of the cell (Fig. 1e,f). However, in the presence 150 of MTS-BioID2-ARL8B-Q75L, GFP-tagged RUFY3.1 and RUFY4 redistributed to mitochondria 151 whereas GFP-tagged RUFY1 and RUFY2 retained their cytosolic/juxtanuclear distribution (Fig. 152 1e,f). In contrast to RUFY3.1-GFP, RUFY3.2-GFP was cytosolic (Supplementary Fig. 1c), 153 probably because it lacks part of the CC2 and FYVE domains (Fig. 1d; see below).

155 To further corroborate these findings, we performed pull-down assays using recombinant GST-

156 ARL8B proteins and FLAG-tagged RUFY proteins expressed by transient transfection in 157 HEK293T cells (Fig. 2a). We observed that GST-ARL8B-Q75L, but not GST-ARL8B-T34N, pulled 158 down both RUFY3.1-FLAG and RUFY4-FLAG (Fig. 2a). In contrast, neither GST-ARL8B protein 159 pulled down RUFY1-FLAG and RUFY2-FLAG (Fig. 2a). Furthermore, we examined the co160 immunoprecipitation of FLAG-tagged RUFY proteins with endogenous ARL8A and ARL8B in 161 HEK293T cells (Fig. 2b). FLAG-tagged forms of the dynein-dynactin adaptor HOOK1 and the 162 ARL8 effector SKIP were used as negative and positive controls, respectively. These 163 experiments showed that RUFY3.1-FLAG and RUFY4-FLAG co-immunoprecipitated 164 endogenous ARL8A and ARL8B, whereas RUFY1-FLAG and RUFY2-FLAG did not (Fig. 2b). Of note, the shorter RUFY3.2 showed negligible co-immunoprecipitation of ARL8A and ARL8B

166 (Fig. 2b).

168 Taken together, these assays demonstrated that both RUFY3.1 and RUFY4 have the ability to 169 interact with GTP-bound, but not GDP-bound, ARL8, suggesting that they behave as bona fide 170 ARL8 effectors. RUFY1 and RUFY2, on the other hand, did not bind to any form of ARL8, ruling 171 out their function as ARL8 effectors. Because RUFY3.2 was cytosolic and did not interact with 172 ARL8, this spliceform was omitted from subsequent experiments, and RUFY3.1 was simply 173 referred to as RUFY3. RUFY4 (also known as ZFYVE31) is expressed at low levels in most 174 tissues and cells, with the exception of the brain, lung and lymphatic organs

175 (https:/ / www.proteinatlas.org/search/rufy4), probably explaining why it was not identified in 
176 the MitoID experiments using HEK293T cells. Nevertheless, because it also behaves as an ARL8

177 effector, we performed some experiments with this protein.

179 The CC2 domain of RUFY3 is required for binding to ARL8

180 We next sought to identify the region of RUFY3 that mediates interaction with ARL8. To this

181 end, we generated deletion constructs of RUFY3-GFP (Fig. 2c) and co-expressed them with

182 MTS-BioID2-ARL8B-Q75L in HeLa cells (Fig. 2d). Interaction with ARL8 was inferred from re-

183 localization of the RUFY3 constructs to mitochondria. By analogy with SKIP, which interacts

184 with ARL8 via the RUN domain (Boucrot et al., 2005; Keren-Kaplan and Bonifacino, 2021; Rosa-

185 Ferreira and Munro, 2011), we expected the homologous RUN domain of RUFY3 to be

186 important. However, we found that deletion of the RUN, CC1 or FYVE domains had no effect

187 on the re-localization of RUFY3-GFP to mitochondria (Fig. 2d,e). Likewisw, combined deletion

188 of the RUN and CC1 domains did not prevent the re-localization of RUFY3-GFP to

189 mitochondria (Fig. 2d,e). However, deletion of the CC2 domain, alone or in combination with

190 the FYVE domain, abrogated re-localization of RUFY3-GFP to mitochondria (Fig. 2d,e).

192 In line with the mitochondrial re-localization experiments, we found that recombinant GST-

193 ARL8B-Q75L pulled down RUFY3-FLAG constructs lacking the RUN, CC1 or FYVE domain

194 expressed in HEK293T cells (Fig. 2f,g). In contrast, GST-ARL8B-Q75L did not pull down

195 RUFY3-FLAG constructs lacking the CC2 domain or the CC2-FYVE tandem (Fig. 2f,g). GST-

196 ARL8B-T34N did not pull down any of the constructs, confirming that the interactions

197 requiring the CC2 domain are exclusive to the GTP-bound form of ARL8B.

199 From these experiments, we concluded that the interaction of RUFY3 with GTP-ARL8B requires

200 the CC2 domain.

202 ARL8B promotes recruitment of RUFY3 and RUFY4 to a juxtanuclear cluster of vesicles

203 We next examined whether RUFY3 and RUFY4 co-localize with ARL8B. Because none of the

204 antibodies we tested detect the endogenous proteins by immunofluorescence microscopy, we

205 examined the localization of fluorescently-tagged versions of the proteins expressed by

206 transient transfection in HeLa cells (Fig. 3a). We observed that both RUFY3-GFP and RUFY4-

207 GFP co-localized with ARL8B-mCherry to a cluster of vesicles adjacent to the nucleus (Fig.

208 3a,b). Double knock out (KO) of ARL8A and ARL8B (ARL8A-B KO) shifted the distribution of

209 RUFY3-GFP and RUFY4-GFP to the cytosol (Fig. 3c). Co-transfection of the ARL8A-B-KO cells 
210 with ARL8B-Q75L restored the association of RUFY3-GFP and RUFY4-GFP with the

211 juxtanuclear vesicle cluster (Fig. 3c).

213 To further dissect the requirement of ARL8 binding for RUFY3 recruitment to vesicles, and the 214 domains of RUFY3 required for this recruitment, we examined the intracellular localization of 215 the RUFY3 deletion mutants depicted in Fig. 2c. We observed that RUFY3 constructs lacking the 216 RUN and/ or CC1 domains were largely associated with the juxtanuclear cluster, whereas those 217 lacking the CC2 or FYVE domains were more cytosolic (Fig. 3d). Combined deletion of the CC2 218 and FYVE domains resulted in a protein that was largely cytosolic (Fig. 3d). Thus, both the 219 ARL8-interacting CC2 domain and the FYVE domain contribute to the localization of RUFY3 to 220 cytoplasmic vesicles.

222 Taken together, the above experiments demonstrated that ARL8 promotes the recruitment of 223 RUFY3 and RUFY4 to a juxtanuclear cluster of vesicles via the CC2 domain. The FYVE domain makes and additional contribution to this recruitment.

\section{RUFY3 and RUFY4 promote retrograde transport of lysosomes}

227 Because ARL8 was previously shown to associate with lysosomes (Bagshaw et al., 2006;

228 Hofmann and Munro, 2006), we examined if the vesicles containing associated RUFY3-GFP and RUFY4-GFP also labeled for the endogenous lysosomal membrane protein LAMP1 in HeLa cells (Fig. 4a). Indeed, we observed significant co-localization of RUFY3-GFP and RUFY4-GFP with LAMP1 (Fig. 4a,b). Moreover, we noticed that cells expressing RUFY3-GFP and RUFY4GFP exhibited more juxtanuclear clustering of lysosomes relative to cells expressing only GFP (Fig. 4c,d). Expression of the different RUFY3-GFP deletion mutants (Fig. 2c) showed that those that bound ARL8 (i.e., constructs lacking the RUN, CC1 or FYVE domains) caused juxtanuclear clustering of lysosomes, whereas those that did not bind ARL8 (i.e., constructs lacking the CC2 domain) failed to cause juxtanuclear clustering (Fig. 4e,f), thus demonstrating a perfect correlation between ARL8 binding and lysosome redistribution by RUFY3 and RUFY4. LAMP1 toward the cell periphery (Fig. 4h-j). RUFY4 mRNA could not be detected by qRT-PCR of HeLa cells (Fig. 4g), consistent with the low expression levels of this mRNA in most cell lines 242 (https: / / www.proteinatlas.org/ENSG00000188282-RUFY4/ celltype). For this reason, the effect of RUFY4 knock down in these cells was not tested. 
245 To determine if the effects of RUFY3 and RUFY4 on the distribution of lysosomes resulted from

246 changes in lysosome transport, we examined the co-localization and movement of vesicles

247 labeled with fluorescently-tagged RUFY3, RUFY4, ARL8B and LAMP1 in the axon and

248 dendrites of rat hippocampal neurons in primary culture, where vesicle movement can be more

249 readily tracked (Fig. 5). We observed that both RUFY3-FLAG and RUFY4-FLAG co-localized

250 with a subpopulation of vesicles containing ARL8B-mCherry and LAMP1-GFP (Fig. 5a,b), as

251 well as the endogenous lysosomal marker LAMTOR4 (Fig. 4c,d), in both the axon and

252 dendrites. We also performed live-cell imaging and kymograph analysis of vesicle movement in

253 the axon, where microtubules are uniformly polarized with their plus ends pointing toward the

254 distal axon. These analyses revealed that RUFY3-GFP and RUFY4-GFP co-moved with LAMP1-

255 RFP, mainly in the retrograde direction (i.e., toward the soma) (Fig. 5e). Moreover, expression of

256 RUFY3-GFP or RUFY4-GFP increased the proportion of retrograde $v s$. anterograde lysosomes

257 (Fig. 5f-h) and caused an overall decrease in the number of moving lysosomes in the axon (Fig.

$2585 \mathrm{~g}, \mathrm{i})$.

260 These experiments thus demonstrated that clustering of lysosomes in the juxtanuclear area of

261 the cell by RUFY3 and RUFY4, is likely due to increased retrograde transport from the cell

262 periphery.

\section{RUFY3 and RUFY4 bind to dynein-dynactin}

The phenotypes resulting from manipulation of RUFY3 and RUFY4 expression are consistent with these proteins playing a role in transport driven by cytoplasmic dynein-dynactin, the only microtubule motor involved in retrograde transport in the cytoplasm (Reck-Peterson et al., 2018). Indeed, we observed that both RUFY3-GFP and RUFY4-GFP co-immunoprecipitated with the endogenous dynein intermediate chain (DIC) and the endogenous $\mathrm{p} 150^{\text {Glued }}$ subunit of dynactin in HEK293T cells (Fig. 6a). In addition, purified, recombinant 6His-GFP-RUFY3 pulled down both endogenous DIC and p150Glued from an extract of HEK293T cells (Fig. 6b). Recombinant 6His-GFP-RUFY4 was degraded and could not be analyzed using this latter assay. Finally, we found that purified, recombinant 6His-GFP-RUFY3 could be pulled down with the purified, recombinant dynein light intermediate chain 1 (DLIC1) and, more specifically, the Cterminal domain of DLIC1 (Fig. 6c), a domain that was previously implicated in interactions with other dynein adaptors (Lee et al., 2018; Vilela et al., 2019). These results thus indicated that RUFY3 and RUFY4 interact with dynein-dynactin, and that the interaction of RUFY3 is direct via the C-terminal domain of DLIC. 
280 To test the functional relevance of interactions of RUFY3 and RUFY4 with dynein-dynactin in

281 cells, we compared the distribution of RUFY3-mCherry and RUFY4-mCherry in the absence or

282 presence of overexpressed GFP-tagged CC1 domain of p150 Glued, a construct that functions as a

283 dominant-negative inhibitor of dynein-dynactin (Quintyne et al., 1999) (Fig. 6d). We observed

284 that, in the absence of GFP-p150 Glued_CC1, RUFY3-mCherry and RUFY4-mCherry localized to a

285 juxtanuclear cluster, whereas in the presence of GFP-p150 Glued_CC1, RUFY3-mCherry and

286 RUFY4-mCherry were associated with peripheral clusters, often found at cell tips (Fig. 6d).

287 These observations demonstrated that interference with dynein-dynactin does not prevent

288 association of RUFY3 and RUFY4 with lysosomes, but precludes their ability to move lysosomes

289 toward the cell center.

291 Targeting of RUFY3 and RUFY4 to peroxisomes promotes their juxtanuclear clustering in a 292 dynein-dependent manner

293 To determine whether RUFY3 and RUFY4 are sufficient for organelle coupling to dynein-

294 dynactin, we next used a peroxisome re-localization assay (Kapitein et al., 2010). Peroxisomes

295 are particularly suited for this assay because they are not very motile. The assay consisted of co-

296 expressing i) a peroxisomal targeting signal from PEX3 (amino acids 1-42) fused to FKBP and

297 RFP, together with ii) RUFY3 or RUFY4 fused to FRB and GFP (Fig. 7a). As a positive control for

298 a known dynein-dynactin adaptor, we used a BICD2 $25-400$-FRB-GFP construct (Fig. 7a). Addition

299 of rapalog brings together the FRB and FKBP domains, leading to the targeting of RUFY3 or

300 RUFY4 to peroxisomes (Fig. 7b). We observed that, in the absence of rapalog, peroxisomes

301 labeled with the PEX3 1-42 -FKBP-RFP construct were scattered throughout the cytoplasm despite

302 the co-expression of RUFY3-FRB-GFP, RUFY4-FRB-GFP or BICD2-FRB-GFP (Fig. 7c and e, -

303 Rapalog). Addition of rapalog, however, resulted in the redistribution of PEX $3_{1-42}$-FKBP-RFP-

304 labeled peroxisomes, together with RUFY3-FRB-GFP, RUFY4-FRB-GFP or BICD2-FRB-GFP, to

305 the juxtanuclear area of the cell (Fig. 7c and e , +Rapalog). In all cases, this redistribution was

306 blocked by knock down of the dynein heavy chain (DHC) (Fig. 7d and e, +Rapalog). These

307 results thus demonstrated that targeting of RUFY3 or RUFY4 to an unrelated organelle is

308 sufficient to promote its redistribution toward the cell center in a dynein-dependent manner.

310 RUFY3 is required for juxtanuclear redistribution of lysosomes upon serum starvation or 311 cytoplasmic alkalinization

312 Removal of serum from the culture medium (i.e., "serum starvation") (Korolchuk et al., 2011; Pu

313 et al., 2017) or cytoplasmic alkalinization (Heuser, 1989) cause redistribution of lysosomes

314 toward the center of the cell. To determine if RUFY3 is required for these processes, we 
315 performed siRNA-mediated knock down of RUFY3 in HeLa cells and examined the effect of 316 serum starvation or alkalinization on the distribution of lysosomes. We observed that both 317 manipulations caused juxtanuclear clustering lysosomes in control cells (Fig. 8a,c), but not in 318 RUFY3-knock down cells (Fig. 8b,c). These experiments thus demonstrated that the function of 319 RUFY3 in mediating dynein-dynactin-dependent transport of lysosomes is required for changes 320 in lysosome positioning in response to specific stimuli. 


\section{Discussion}

322

At steady state, lysosomes exhibit a characteristic distribution, consisting of a densely packed population in the juxtanuclear area and a scattered population in the periphery of the cell (reviewed by (Ballabio and Bonifacino, 2020; Bonifacino and Neefjes, 2017). In polarized cells such as neurons, the peripheral population of lysosomes includes distinct pools in specialized domains of the cells (e.g., axon and dendrites) (De Pace et al., 2020; Farfel-Becker et al., 2019; Farias et al., 2017; Lee et al., 2011; Tsuruta and Dolmetsch, 2015). The overall distribution of lysosomes results from the integration of various processes, including tethering to other organelles such as the endoplasmic reticulum (ER) (Jongsma et al., 2016; Raiborg et al., 2015; Rocha et al., 2009; Saric et al., 2021) and mobilization by coupling to microtubule motors (Harada et al., 1998; Hollenbeck and Swanson, 1990). Transport of lysosomes toward microtubule plus ends (i.e., anterograde transport) or minus ends (i.e., retrograde transport) depends on coupling to kinesin or dynein-dynactin motors, respectively (Harada et al., 1998; Hollenbeck and Swanson, 1990) (Fig. 8d). Coupling to both types of motor is not direct but mediated by small GTPases, adaptors and other effectors and regulators (Ballabio and Bonifacino, 2020; Bonifacino and Neefjes, 2017). Since there is only one cytoplasmic dynein (in contrast to the $\sim 45$ kinesins encoded in mammalian genomes), multiple combinations of adaptors and regulators allow coupling of dynein-dynactin not only to different organelles, but also to the same organelle with different functional properties. The multiple systems shown to couple lysosomes to dynein-dynactin include the small GTPase RAB7 and its effector RILP (Jordens et al., 2001), the transmembrane protein TMEM55B and adaptor protein JIP4 (Gowrishankar et al., 2021; Willett et al., 2017) , the related adaptor protein JIP3 (Drerup and Nechiporuk, 2013; Gowrishankar et al., 2021), the calcium channel MCOLN1 and penta-EFhand protein ALG2 (Li et al., 2016), the septin protein SEPT9 (Kesisova et al., 2021), and the BLOC-1/BORC component SNAPIN (Cai et al., 2010). In the present study, we identify RUFY3 and RUFY4 as novel ARL8 effectors that couple lysosomes to dynein-dynactin (Fig. 8d).

Previous studies had characterized a short, 469-amino-acid form of RUFY3 (denoted here as RUFY3.2) that lacks part of the CC2 domain and the entire FYVE domain present in the predicted long, 620-amino-acid form of the protein (RUFY3.1) (Fig. 1d). Both forms arise by alternative splicing of the RUFY3 pre-mRNA. The short form had been shown to be particularly abundant in the brain, and to play roles in neuronal polarity and the regulation of axon specification, growth and degeneration (Hertz et al., 2019; Honda et al., 2017; Mori et al., 2007; Wei et al., 2014). The existence, distribution and function of the long form had not been 
356 previously documented. Our MitoID procedure using ARL8A and ARL8B as baits identified RUFY3, including peptides only found in the longer RUFY3.3 form, as a top hit. This finding thus demonstrated for the first time that the longer form exists and is expressed in nonneuronal cells. Together with expression data from the Human Protein Atlas

360 (https: / / www.proteinatlas.org/search/ rufy3), the isolation of RUFY3.1 from HEK293T cells is consistent with the involvement of RUFY3 in non-neuronal processes such as migration, invasion and metastasis of lung, gastric and colorectal cancer cells (Men et al., 2019; Wang et al., 2015; Xie et al., 2017; Zhu et al., 2019).

The 571-amino-acid RUFY4 protein had been previously shown to be expressed mainly in lung and lymphatic organs, as well as in dendritic cells and macrophages (Men et al., 2019). The Human Protein Atlas also reports detectable expression of the RUFY4 mRNA in the brain, gastrointestinal tract and prostate (https: / / www.proteinatlas.org/ search/rufy4), but very low levels in other tissues and cells. Functional studies revealed roles of RUFY4 in autophagosome formation, autophagosome-lysosome fusion and degradation of autophagic substrates such as damaged mitochondria and intracellular bacteria in phagocytic cells (Lassen et al., 2016;

372 Terawaki et al., 2015).

Our findings suggest that the functions of RUFY3 in neurons and cancer cells, and RUFY4 in phagocytic cells, might be related to the ability of these proteins to couple lysosomes to dyneindynactin. Indeed, processes such as the regulation of axonal functions (Adnan et al., 2020; Farias et al., 2017; Palomo-Guerrero et al., 2019), cancer cell migration, invasion and metastasis (Dykes et al., 2016; Schiefermeier et al., 2014; Steffan et al., 2014), and autophagy (Farias et al., 2017; Jia et al., 2017; Korolchuk et al., 2011; Marwaha et al., 2017) have all been shown to be influenced by lysosome positioning and motility, consistent with a role for RUFY3 and RUFY4 in the regulation of lysosomal functions.

Further analyses demonstrated that both RUFY3 and RUFY4 interact with the GTP-bound form of ARL8 and are recruited to lysosomes in an ARL8-dependent manner. Although ARL8 was previously shown to bind to the RUN domains of SKIP and PLEKHM1 (Farias et al., 2017; Keren-Kaplan and Bonifacino, 2021; Marwaha et al., 2017; Rosa-Ferreira and Munro, 2011), we find that binding of ARL8 to RUFY3 involves the CC2 domain of RUFY3. These observations imply that ARL8 can bind its effectors by different mechanisms. We did not dissect the ARL8binding site on RUFY4, but there is homology to RUFY3 in the CC2 region, making it likely that both proteins interact with ARL8 in a similar manner. 
392 Mutational dissection of RUFY3 showed that the FYVE domain also contributes to the

393 association of RUFY3 with lysosomes. It remains to be established, however, how the RUFY3

394 FYVE domain contributes to these functions, since it lacks the tandem histidine residue cluster required for binding to PtdIns(3)P on endolysosomal membranes (Char and Pierre, 2020).

Despite having homology to RUFY3 in the CC2 domain and other domains, RUFY1 and RUFY2 did not interact with ARL8. Instead, RUFY1 was previously shown to interact with the small GTPases RAB4, RAB5, and RAB14, and to regulate various early endosomal functions (Cormont et al., 2001; Gosney et al., 2018; Nag et al., 2018; Vukmirica et al., 2006). RUFY2, on the other hand, was shown to interact with the Golgi complex-associated small GTPase RAB33A, which

402 functions in autophagosome formation (Fukuda and Itoh, 2008; Fukuda et al., 2011). These

403 interactions and functions are consistent with the differences in association of ARL8 with different RUFY family members.

In both HeLa cells and rat hippocampal neurons, transgenic RUFY3 or RUFY4 constructs colocalized with ARL8 and LAMP1 on lysosomes. Moreover, overexpression of RUFY3 or RUFY4 constructs in HeLa cells caused juxtanuclear clustering of lysosomes, and in neurons increased retrograde transport and caused depletion of lysosomes from the axon. These effects are in line with a role for RUFY3 and RUFY4 as dynein-dynactin adaptors demonstrated here. The effects in axonal transport are also in accordance with the previously reported roles of RUFY 3 in neurons (Hertz et al., 2019; Honda et al., 2017; Mori et al., 2007; Wei et al., 2014). Importantly,

413 knock down of RUFY3 in HeLa cells resulted in dispersal of lysosomes toward the cell

414 periphery, also as expected for a dynein-dynactin adaptor. RUFY3 knock down also prevented

415 the juxtanuclear clustering of lysosomes induced by serum starvation or cytoplasmic

416 alkalinization. These findings indicate that RUFY3 is required for maintenance of the juxtanuclear population of lysosomes at steady-state, and for the repositioning of the peripheral population of lysosomes to the cell center in response to specific stimuli.

These findings are surprising in light of the many other proteins that were previously shown to couple lysosomes to dynein-dynactin. Why are so many dynein-dynactin adaptors involved in this process? Once possibility is that they all contribute to the overall strength of coupling. The absence of any of these adaptors could weaken the interactions of lysosomes with dyneindynactin, tilting the balance toward interactions with kinesins and thus shifting the distribution of lysosomes to the cell periphery. The different dynein-dynactin adaptors could also have celltype specific functions, depending on their relative expression levels. In addition, the various dynein-dynactin adaptors could be differentially regulated in response to specific stimuli, as 
428 would be expected from their interactions with different GTPases and calcium-binding proteins. 429 Furthermore, the adaptors could be associated with different populations of lysosomes. For 430 simplicity, in this study we use "lysosomes" as an all-encompassing term for a variety of 431 LAMP1-positive endolysosomal organelles. However, it is well known that LAMP1-positive 432 organelles include functionally distinct populations of lysosomes (Johnson et al., 2016; Vukoja et 433 al., 2018), late endosomes and even some early endosomes (Fermie et al., 2018; Saric et al., 2021).

434 In this regard, RUFY3/4 and RILP function as dynein-dynactin adaptors for populations of 435 endolysosomes decorated with ARL8 and RAB7, respectively (Jongsma et al., 2020). Finally, 436 different dynein-dynactin adaptors could participate in a sequential handoff mechanism, as 437 recently reported for the retrograde transport of maturing autophagosomes in the axon (Cason 438 et al., 2020).

Another conundrum that remains to be solved is how ARL8 can regulate both anterograde 441 lysosome transport of lysosomes through recruitment of kinesin-1 and kinesin-3 (Guardia et al., 442 2016; Rosa-Ferreira and Munro, 2011), and retrograde lysosome transport through recruitment 443 of dynein-dynactin (this study) (Fig. 8c). Studies in Drosophila also showed that ARL8 can 444 interact with the ortholog of RILP, a known dynein-dynactin interacting protein (Rosa-Ferreira 445 et al., 2018). This regulation of opposing processes by the same GTPase is not exclusive to ARL8, 446 though, since RAB7 also promotes anterograde lysosome transport via FYCO1 (Raiborg et al., 447 2015) and retrograde lysosome transport via RILP (Jordens et al., 2001). For both GTPases, there 448 must be other regulators that determine the interaction with alternative adaptors and, 449 consequently, the direction of lysosome transport. Nevertheless, the role of ARL8 in 450 anterograde transport seems to be dominant over that in retrograde transport, since depletion of ARL8 or its positive regulator BORC cause juxtanuclear clustering of lysosomes, whereas overexpression of ARL8 drives lysosomes to the cell periphery (De Pace et al., 2020; Farias et al., 2017; Guardia et al., 2016; Keren-Kaplan and Bonifacino, 2021; Korolchuk et al., 2011; Pu et al., 2015; Rosa-Ferreira and Munro, 2011). Future studies will have to address under what conditions ARL8 promotes lysosome retrograde transport mediated by RUFY3 and RUFY4.

457 Our experiments using all recombinant proteins have shown that RUFY3 has the ability to bind 458 directly to the C-terminal domain of DLIC1, a property shared with other dynein-dynactin 459 adaptors such as BICD2, SPDL1 and HOOK1-3 (Reck-Peterson et al., 2018). As for these 460 adaptors, the interactions could involve coiled-coil regions such as the RUFY3 CC2 domain. 461 However, we cannot rule out the possibility that RUFY3 and RUFY4 also exert their functions 462 indirectly, through interactions with other dynein-dynactin adaptors, perhaps providing an 463 additional or alternative anchorage to lysosomes via ARL8. Further studies will be needed to 
464 elucidate how the function of multiple lysosomal dynein-dynactin adaptors is integrated and

465 how these functions are coordinated with those of kinesin adaptors to control the dynamic

466 distribution of lysosomes under different physiological conditions.

\section{Acknowledgements}

469 We thank for Xiaolin Zhu and Boma Fubara excellent technical assistance, Anna Akhmanova, 470 Brett Collins, Wade Harper, Steve Jackson, Walter Mothes, Kyle Roux and Ron Vale for kind 471 gifts of reagents, and other members of the Bonifacino lab for helpful discussions and support. 472 This work was supported by the Intramural Program of NICHD, NIH (project \# ZIA

473 HD001607).

\section{Author contributions}

476 T.K.K and J.S.B conceived the project. T.K.K designed and conducted most of the experiments.

477 A.S. contributed reagents, conducted shell analysis of lysosome distribution and qRT-PCR. S.G.

478 conducted and analyzed experiments in neurons. C.W. contributed to live-cell imaging and data 479 quantification. R.J helped with experiments of lysosome repositioning. Y.L conducted mass 480 spectrometry. T.K.K and J.S.B wrote the manuscript with input from all authors. 


\section{Recombinant DNAs}

Mitochondrially-targeted ARL8 (mito-ARL8) constructs were created as follows: DNA sequences encoding the mitochondrial-targeting sequence of human TOM20 (amino acids 1-30, MVGRNSAIAAGVCGALFIGYCIYFDRKRRS) (Kanaji et al., 2000), followed by a short GAGA linker, were inserted into the pcDNA3.1-myc-BioID2-MCS plasmid (Kim et al., 2016) (a gift from Kyle Roux, Addgene \#74223) by PCR to create pcDNA3.1-TOM20-myc-BioID2. Next, DNA sequences encoding human ARL8A or ARL8B lacking the N-terminal helix (amino acids 1-17) and harboring the Q75L or T34N mutations, and an N-terminally GAGA linker, were inserted into the XhoI and BamHI sites of pcDNA3.1-TOM20-myc-BioID2. The resulting plasmids encoded TOM20-GAGA-myc-BioID2-GAGA-ARL8 fusion proteins. Plasmids encoding RUFY3 deletion mutants were generated by KLD mutagenesis (Cat\# M0554S, New England Biolabs) on the backbone of RUFY3-GFP and RUFY3-FLAG plasmids. The plasmid pcDNA3.1-SKIP-FOS was generated by insertion of SKIP coding sequences into the $\mathrm{XbaI}$ and KpnI sites of pcDNA3.1-FOS (FLAG-One-Strep). DNA sequences encoding the peroxisometargeting sequence of PEX3 (amino acids 1-42) were cloned by KLD mutagenesis into the

RUFY isoforms used in the study: RUFY1 isoform 1, NM_025158.5; RUFY2 isoform 1, NM_017987.4; RUFY3 isoform 1 (RUFY3.1), NM_001037442.4; RUFY3 isoform 2 (RUFY3.2), NM_014961.5; RUFY4 isoform 1, NM_198483.3. pcDNA3.1+/C-(K)-DYK-RUFY-FLAG plasmids OHu19866D, OHu02933D, OHu24594D, OHu24610D, OHu55786D, respectively, were purchased from GenScript Biotech. These plasmids were used to create plasmids encoding RUFY-GFP and RUFY-mCherry constructs by amplifying RUFY coding sequences and inserting them into EcoRI-digested pEGFP-N1 and pmCherry-N1 plasmids, respectively, by Gibson

To create a pEGFP-N1-RUFY3-FRB-EGFP, a pEGFP-N1-SKIP ${ }_{1-300}$-FRB-EGFP plasmid was

512 digested with SalI and AgeI and the fragment containing the FRB coding sequence was cloned

513 into pEGFP-N1-RUFY3-GFP digested with the same enzymes. To create a pEGFP-N1-RUFY4-

514 FRB-EGFP plasmid, pEGFP-N1-SKIP ${ }_{1-300}$-FRB-EGFP was digested with XhoI and SalI and the

515 fragment containing the FRB coding sequence was cloned into pEGFP-N1-RUFY4-EGFP

516 digested with the same enzymes. A BICD2 fragment encoding amino acids 25-400 was 
517 amplified by PCR from pEGFP-N1-PEX3*-SBP-GFP (Guardia et al., 2019) (Addgene \#120174),

518 digested with BamHI and SalI, and ligated into pEGFP-N1-SKIP ${ }_{1-300}$-FRB-EGFP digested with

519 BglII and SalI. To create pET28a-6His-sfGFP-RUFY3.1, the coding sequences of RUFY3.1 were

520 amplified by PCR and inserted into KpnI and NotI double-digested pET28a-6His-sfGFP-BICD2

521 (to replace the BICD2 with RUFY3) by Gibson assembly. To creat the pEGFP-C1-p150 ${ }^{\text {Glued_CC1 }}$

522 the region encoding CC1 (amino acids 205-540) domain from chicken was cloned into the

523 pEGFP-C1 plasmid between and EcoRI and SalI sites.

Other plasmids used in our study were: pMSCV-N-HA-FLAG-HOOK1 (gift from Wade Harper), pLAMP1-RFP (gift from W. Mothes Addgene \#1817), pEGFP-N1-LAMP1-EGFP (Farias et al., 2017) and pEGFP-C1-FLAG (Britton et al., 2013) (a gift from Steve Jackson, Addgene\# 46956), GFP-BICD2 (a gift from Anna Akhmanova), pET28a-6His-sfGFP-BICD2, GST-DLIC1 and GST-DLIC1-CT (gifts from Ron Vale (Schroeder et al., 2014), pOPINE-GFPnanobody (Kubala et al., 2010) (a gift from Brett Collins, Addgene \#49172). All plasmids sequences were verified by Sanger sequencing (Genewiz or Eurofins Genomics).

\section{Cell culture and treatments}

534 HeLa and HEK293T cells (ATCC) were maintained in Dulbecco's Modified Eagle's Medium (DMEM) (Cat\# 112-319-101, Quality Biological) with 10\% fetal bovine serum (35-011-CV,

536 Corning), $50 \mathrm{U} / \mathrm{mL}$ penicillin, $50 \mu \mathrm{g} / \mathrm{mL}$ streptomycin (Cat\# 30002-CL, Corning) (CDMEM) and 537 incubated in $5 \% \mathrm{CO}_{2}$ and $37^{\circ} \mathrm{C}$. Lipofectamine 2000 (Cat\# 11668019, Thermo Fisher) was used 538 for transfections according to manufacturer's protocol. Briefly, for immunofluorescence 539 microscopy and live-cell imaging, 0.1-0.5 $\mu \mathrm{g}$ plasmid with $1 \mu \mathrm{l}$ lipofectamine was used for 540 transfection in 24-well and live-cell imaging chambers. Transfection mixture in Opti-MEM 541 (Cat\# 31985070, Gibco) was added to wells with fresh CDMEM. Culture medium was replaced 542 by CDMEM $1 \mathrm{~h}$ after transfection. Cells were fixed or imaged $\sim 24 \mathrm{~h}$ after transfection. For co543 immunoprecipitation experiments, 1-8 $\mu$ g plasmid DNA and $25 \mu \mathrm{l}$ lipofectamine were used per $54410 \mathrm{~cm}$ plate. $3 \mathrm{~mL}$ transfection mixture in Opti-MEM was added to plates containing $12 \mathrm{~mL}$ fresh CDMEM. Cells were harvested $\sim 24 \mathrm{~h}$ after transfection.

547 The following siRNAs were used in this study: non-targeting siRNA (5'-

548 UGGUUUACAUGUCGACUAAUU-3' (Dharmacon) (labeled with phosphate at the 5'), ON549 TARGETplus Human RUFY3 siRNA SMARTpool (Cat\# L-020336-00-0005, Horizon Discovery), 550 Silencer Select siRNA to DYNC1H1 (ID: s4200, Cat\# 4390824, Thermo Fisher). siRNA treatments 551 were done with Oligofectamine (Cat\# 12252011, Thermo Fisher) according to manufacturer's 
552 protocol. Briefly, $2.5 \mu \mathrm{l}$ of $20 \mu \mathrm{M}$ siRNA was used per 24-well plate, or $10 \mu \mathrm{l}$ per 6-well plate. For

553 Fig. 4h, one shot of siRNA was used in a 48 h treatment. For Fig. 7, cells were treated with one

554 shot of siRNA and transfected with plasmids $24 \mathrm{~h}$ after the siRNA shot. The peroxisome

555 motility assay was carried out $24 \mathrm{~h}$ after transfection (a total of $48 \mathrm{~h}$ siRNA treatment). The cells

556 were treated with or without $0.5 \mu \mathrm{M}$ rapalog (Cat\# 635057, Takara Bio) for $1 \mathrm{~h}$. For siRNA

557 experiments in Fig. 8a,b, HeLa cells were treated with the siRNAs for $96 \mathrm{~h}$ ( 2 shots of siRNA).

558 Serum starvation was performed by incubating cells in serum-free DMEM for $1 \mathrm{~h}$ at $37^{\circ} \mathrm{C}$.

559 Alkaline medium treatment was performed by incubating cells in complete DMEM adjusted to

$560 \mathrm{pH} 8.5$ with $\mathrm{NaOH}$ for $1 \mathrm{~h}$ at $37^{\circ} \mathrm{C}$. After incubation, cells were fixed with $4 \%$ paraformaldehyde

561 for $15 \mathrm{~min}$ at room temperature and processed for immunofluorescence microscopy.

562

563 Cover slips and live-cell chambers were pre-coated with fibronectin ( Cat\#F2006, Millipore-

564 Sigma). The following plates were used in the study: 4- and 8-well live-cell chambers (Cat\# C4-

565 1.5H-N, Cat\# C8-1.5H-N, Cellvis), 10-cm plates (Cat\# 353003, Corning), 15-cm plates (Cat\#

566 353025, Corning) and 24-well plates (Cat\# 353047, Corning).

568 Identification of ARL8-interacting proteins by MitoID

569 ARL8 interacting proteins were identified by MitoID (Gillingham et al., 2019; Roux et al., 2013)

570 with modifications. HEK293T cells $\left(5.4 \times 10^{6}\right)$ were plated on 15-cm plates (Cat\# 353025,

571 Corning). The next day, cells were transfected with $50 \mu$ l Lipofectamine 2000 (Cat\# 11668019,

572 Thermo Fisher Scientific) and $25 \mu \mathrm{g}$ plasmid encoding Mito-ARL8 constructs and Mito-BioID2

573 as a negative control (Fig. 1a). We prepared two 15-mL tubes with Opti-MEM; one was mixed

574 with the DNA and the second with Lipofectamine 2000. After 5-min incubation at room

575 temperature, the contents of the tubes were combined, and the mix incubated at room

576 temperature for an additional $20 \mathrm{~min}$. The $6 \mathrm{~mL}$ mix was added to the plates containing the cells

577 that were filled with $24 \mathrm{~mL}$ of fresh, prewarmed CDMEM supplemented with MycoZap Plus-

578 CL (Cat\# VZA-2011, Lonza). At $22 \mathrm{~h}$ after transfection, $50 \mu \mathrm{M}$ biotin (Cat\# 47868, Millipore-

579 Sigma) was added to each plate ( $1.5 \mathrm{~mL}$ from $1 \mathrm{mM}$ stock). $24 \mathrm{~h}$ after biotin addition, cells were

580 scraped from the plate with $4 \mathrm{~mL}$ cold PBS and washed 3 times with centrifugation for $5 \mathrm{~min}$, at

$5814{ }^{\circ} \mathrm{C}, 500 \times g$. Cell pellets were kept at $-80^{\circ} \mathrm{C}$. Two plates were used for each condition. The

582 experiment was done with 3 biological replicates and all samples were processed

583 simultaneously. Thawed cells were resuspended in $5 \mathrm{~mL}$ buffer A (25 mM Tris- $\mathrm{HCl}$ pH 7.4, 150

$584 \mathrm{mM} \mathrm{NaCl}, 1 \mathrm{mM}$ EDTA $1 \%$ Triton X-100) supplemented with protease inhibitor tablet (Cat\#

585 1836170, Roche). The two plates corresponding to the same condition were combined at this 
586 stage and incubated for $1 \mathrm{~h}$, at $4{ }^{\circ} \mathrm{C}$ with gentle rotation. The soluble fraction was separated by centrifugation for $20 \mathrm{~min}$ at $4^{\circ} \mathrm{C}, 17,000 \mathrm{xg}$. A neutravidin-agarose slurry (Cat\# 29201,

588 Pierce $^{\mathrm{TM}}$ NeutrAvidin ${ }^{\mathrm{TM}}$ Agarose) (500 $\mu \mathrm{l}$, corresponding to $250 \mu \mathrm{l}$ beads) was washed in $14 \mathrm{~mL}$

589 buffer $\mathrm{A}$. The supernatant was incubated with the neutravidin-agarose overnight at $4{ }^{\circ} \mathrm{C}$ with

590 gentle rotation. The beads were separated from the lysate by centrifugation for $5 \mathrm{~min}$ at $500 \times \mathrm{g}$

591 and $4{ }^{\circ} \mathrm{C}$, and washed twice in $3 \mathrm{~mL}$ buffer B (2\% SDS), 3 times in $5 \mathrm{~mL}$ buffer C $(0.1 \%$

592 deoxycholic acid, 1\% Triton X-100, 1 mM EDTA, $0.5 \mathrm{M} \mathrm{NaCl}, 50 \mathrm{mM} \mathrm{HEPES} \mathrm{pH} \mathrm{7.5),} \mathrm{and} \mathrm{once}$

593 in $5 \mathrm{~mL} 50 \mathrm{mM}$ Tris- $\mathrm{HCl} \mathrm{pH} 7.4,50 \mathrm{mM} \mathrm{NaCl}$. Between washes, samples were centrifuged for 5

$594 \min$ at $4{ }^{\circ} \mathrm{C}, 500 \times g$. Lastly, the washed neutravidin-agarose was resuspended in $75 \mu \mathrm{l} 4 \mathrm{X}$

595 Laemmli buffer (Cat\# 1610747, Bio-Rad) and samples were heated for $10 \mathrm{~min}$ at $99{ }^{\circ} \mathrm{C} .60 \mu \mathrm{l}$

596 were loaded onto 12\% TGX precast gels (Cat\# 4561043, Bio-Rad), which were run for a few

597 minutes to allow the sample to enter the gel.

\section{Mass spectrometry}

600 Bands containing the entire sample were cut from the gel. Samples were reduced with $10 \mathrm{mM}$

601 TECP for $1 \mathrm{~h}$, alkylated with $10 \mathrm{mM}$ NEM for $10 \mathrm{~min}$, and digested with trypsin at 37 oC over-

602 night. Peptides were extracted from the gel and desalted using Oasis HLB $\mu$ Elution plates

603 (Waters). Digests of each sample were injected into an Ultimate 3000 RSLC nano HPLC system

604 (Thermo Fisher). Peptides were separated on an ES802 column over a 63-min gradient with

605 mobile phase B (98\% acetonitrile, $1.9 \% \mathrm{H}_{2} \mathrm{O}, 0.1 \%$ formic acid) increased from $3 \%$ to $24 \%$. LC-

606 MS / MS data were acquired on an Orbitrap Lumos mass spectrometer (Thermo Fisher

607 Scientific) in data-dependent acquisition mode. The MS1 scans were performed in Orbitrap with

608 a resolution of $120 \mathrm{~K}$, a mass range of $375-1500 \mathrm{~m} / \mathrm{z}$, and an AGC target of $2 \times 10^{5}$. The

609 quadrupole isolation was used with a window of $1.5 \mathrm{~m} / \mathrm{z}$. The MS/MS scans were triggered

610 when the intensity of precursor ions with a charge state between 2 to 6 reached $1 \times 10^{4}$. The MS2

611 scans were conducted in ion trap. The CID method was used with collision energy fixed at 30\%.

612 The instrument was run in top speed mode. MS1 scan was performed every 3 sec, and as many

613 MS2 scans were acquired within the 3 sec cycle. Database search and label-free quantification

614 were performed using Proteome Discoverer 2.2 software. Up to 2 missed cleavages were

615 allowed for trypsin digestion. NEM on cysteines and oxidation on methionine were set as fixed

616 and variable modifications, respectively. Mass tolerances for MS1 and MS2 scans were set to 5

617 ppm and 0.6 Da, respectively. The search results were filtered by a false discovery rate of $1 \%$ at

618 the protein level. The summed intensity of the unique peptides was used for protein ratio

619 calculation. The missing values were imputed. The maximum and minimum fold changes

620 allowed were set to 100 and 0.01 respectively. The total peptide amount of each sample was 
621 used for normalization. The individual protein ANOVA method was used for hypothesis test.

622 Proteins with $\log 2$ fold change $\geq 1$ or $\leq-1$, and adjusted $\mathrm{p} \leq 0.05$ were considered significantly

623 changed.

\section{Antibodies}

626 Primary antibodies: FLAG-HRP (Cat\# A8592, RRID:AB_439702, mouse, 1:5,000-1:6,000,

627 Millipore-Sigma), ARL8A (Cat\# 17060-1-AP, RRID:AB_2058998, rabbit, 1:500, Proteintech),

628 ARL8B (Cat\# C13049-1-AP, RRID:AB_2059000, rabbit, 1:500, Proteintech), TOM20 (Cat\# 11802-

629 1-AP, RRID:AB_2207530, rabbit, 1:500, Proteintech), BioID2 (Cat\# BID2-CP-100, chicken, 1:2000,

630 BioFront Technologies), p150-glued (Cat\# 610473, RRID:AB_397845, mouse, 1:300, BD

631 Biosciences), DIC (Cat\# MAB1618, RRID: AB_224605, mouse,1:200, Millipore-Sigma),

632 Streptavidin-HRP (Cat\# 21130, 1:10,000, Pierce), GFP-HRP (Cat\# 130-091-833, RRID:AB_247003

633 mouse, 1:2,000, Miltenyi Biotec), LAMTOR4 (C7orf59) (D4P6O) (Cat\# 13140, RRID:AB_2798129,

634 rabbit, 1:200, Cell Signaling Technology), LAMP1 ([H4A3], DSHB Hybridoma Product H4A3,

635 mouse, 1:500 deposited by J.T. August and J.E.K. Hildreth). FLAG (Cat\# F1804, mouse, 1:200,

636 Millipore-Sigma Sigma).

638 Secondary antibodies: HRP-conjugated goat anti-rabbit IgG (H+L), (Cat\# 111-035-003,

639 RRID:AB_2313567, 1:10,000 Jackson ImmunoResearch), HRP-conjugated donkey anti-mouse

640 IgG (H+L) (Cat\# 715-035-150, RRID:AB_2340770,1:10,000 Jackson ImmunoResearch), donkey-

641 anti-mouse IgG Alexa Fluor 488 (Cat\# A21202, 1:2,000, Thermo Fisher), donkey-anti-mouse IgG

642 Alexa Fluor 555 (Cat\# A31570, 1:2000, Thermo Fisher), Alexa Fluor 546-phalloidin (Cat\#

643 A22283, 1:2000, Thermo Fisher), goat anti-Chicken IgY (H+L) Alexa Fluor 555 (Cat\# A21437,

644 1:1000, Thermo Fisher), donkey anti-mouse IgG Alexa Fluor 647 (Cat\# A31571,

645 RRID:AB_162542, 1:1,000, Thermo Fisher)

\section{Immunofluorescence microscopy}

648 Cells were washed 3 times with PBS, fixed with $4 \%$ paraformaldehyde (PFA) for 15 min at 649 room temperature, washed 3 times with PBS, incubated with PBS supplemented with $0.1 \%$ 650 saponin and 0.5-1\% BSA for $30 \mathrm{~min}$ at room temperature (blocking buffer), incubated with 651 primary antibodies that was diluted in blocking buffer, for $30 \mathrm{~min}$ at $37^{\circ} \mathrm{C}$, washed 3 times with 652 PBS, incubated with secondary antibodies diluted in blocking buffer for $30 \mathrm{~min}$ at $37^{\circ} \mathrm{C}$, washed 653 twice with PBS and once with distilled water, and mounted on slides using Fluoromount-G 654 with DAPI (Cat\# 0100-20, Electron Microscopy Sciences). Alexa Fluor 546-phalloidin was added 
655 for $15 \mathrm{~min}$ at room termpature, after secondary antibody was removed and coverslip was

656 washed 3 time in PBS.

\section{Image acquisition}

659 Images were acquired on a Zeiss LSM780 or Zeiss LSM880 inverted confocal laser scanning 660 microscopes fitted with a Plan-Apochromat 63X, 1.4 numerical aperture (NA) objective (Carl 661 Zeiss). Live-cell imaging was performed in a controlled chamber $\left(37^{\circ} \mathrm{C}\right.$ and $\left.5 \% \mathrm{CO}_{2}\right)$. Z-stacks

662 were obtained, and maximal intensity projections were generated. Images were further

663 processed in ImageJ (Schneider et al., 2012).

\section{Lysosome positioning measurements}

666 To quantify lysosome positioning (Figs. 4d,f,i,j and 8c), we applied a "shell analysis"(Saric et al., 667 2021). Briefly, z-stack confocal fluorescence micrographs of cells immunostained for LAMP1 668 were flattened and a threshold was applied to eliminate background. Cells with a relatively 669 centered nucleus and uniform shape were selected for the analysis, as narrow, elongated cells,

670 could not be accurately quantified. These criteria were pre-defined and applied to all 671 conditions. Cells meeting these criteria were manually traced in ImageJ/Fiji using either

672 cytosolic GFP signal or phalloidin-stained cortical actin for visualization. The total area 673 corresponding to LAMP1 signal in the cell was measured. Then, the cell outlines were 674 consecutively reduced in size by a fixed length a total of 5 times, and the LAMP1 area scored each time. Such an approach resulted in 5 shells within the cell with shell 1 covering the cell vertices and shell 5 the perinuclear region. The LAMP1 signal area within shell 5 was calculated as a percentage of total LAMP1 area to give the percent perinuclear LAMP1 signal. The LAMP1 signal area within shell 1 was calculated as a percentage of total LAMP1 area to give the percent peripheral LAMP1 signal (Fig. 4j).

\section{Co-localization analysis}

683 Co-localization analysis (Fig.3c,4b) was done using the Pearson-Spearman correlation (PSC) 684 plug-in for ImageJ/ Fiji (Schneider et al., 2012) Scatter plots of co-localization report the 685 Pearson's correlation coefficient (French et al., 2008), representing the relationship of the signal 686 intensity from green (overexpression of GFP or GFP-RUFY constructs) and red (endogenously 687 labeled LAMP1 or ARL8B-mCherry) channels of analyzed images. This value can range from -1 688 to +1 , where 0 indicates no relationship and -1 and +1 indicate strong negative or positive 689 correlation, respectively. The plugin allowed masking of areas to be included in the analysis. In 
690 a given image, individual cells were masked prior to analysis using the selection brush tool as

691 described (French et al., 2008) to determine the Pearson's correlation coefficient per cell of GFP

692 and LAMP1 or ARL8B-mCherry signals. A threshold level of 10 was set, under which pixel

693 values were considered noise and not included in the statistical analysis. Three experimental

694 replicates were done. The mean Pearson's correlation coefficient per cell from each replicate

695 experiment was plotted, and statistical significance between conditions was determined using

696 one-way ANOVA or unpaired Student's t-test with multiple comparisons to the GFP control

$697(\mathrm{n}=3)$.

\section{Manual scoring of microcopy experiments}

700 Scoring of cells in which RUFY proteins localized to mitochondria (Figs. 1f, 2e) was done by

701 visually scoring cells based on the RUFY-GFP signal (a minimum of 300 cells per condition from

702 a total of 3 independent experiments). Scoring of peroxisome distribution (Fig. 7e) was done by

703 visually scoring cells based on the peroxisome phenotype that was detected by the RFP signal of

704 the PEX3 ${ }_{1-42}$-FKBP-RFP plasmid for juxtanuclear, partially juxtanuclear and dispersed

705 peroxisomes (a minimum of 300 cells per condition from a total of 3 independent experiments

706 were scored, except for the BICD2 construct in the NT siRNA +Rapalog condition in which 200

707 cells from 2 experiments were used for the analysis).

709 Co-immunoprecipitation

$7102.5 \times 10^{6} \mathrm{HEK} 293 \mathrm{~T}$ cells were plated on 10-cm dishes and transfected the following day.

711 Following transfection, cells were scraped and washed 3 times in cold PBS for 5 min at $4{ }^{\circ} \mathrm{C}$ with

712 a $500 \times g$ spin between washes. Cell pellets were resuspended in $1 \mathrm{~mL}$ cold lysis buffer and

713 incubated for $30 \mathrm{~min}$ at $4{ }^{\circ} \mathrm{C}$ with gentle rotation. In Fig. 2b, buffer composition was: $25 \mathrm{mM}$

714 Tris-HCl pH 7.4, 0.15 M NaCl, 1 mM EDTA, 1\% NP-40 (Cat\# 011332473001, Roche), $5 \%$

715 glycerol, supplemented with complete EDTA-free protease inhibitor capsule (Cat\# 1836170,

716 Roche). Following lysis, the soluble fraction was separated by centrifugation for $10 \mathrm{~min}$ at $4{ }^{\circ} \mathrm{C}$,

717 17,000 x g. Lysates were incubated on $20 \mu \mathrm{l}$ magnetic-FLAG-agarose (Cat\# A36797, Thermo

718 Fisher) overnight at $4{ }^{\circ} \mathrm{C}$ with gentle rotation. Following incubation, cells were washed 3 times

719 in $1 \mathrm{~mL}$ of $25 \mathrm{mM}$ Tris- $\mathrm{HCl} \mathrm{pH} 7.5,150 \mathrm{mM} \mathrm{NaCl}, 0.05 \%$ Tween-20 for $5 \mathrm{~min}$ at $4{ }^{\circ} \mathrm{C}$, with a 500

$720 \times g$ spin between washes. Washed beads were eluted by addition of Laemmli sample buffer and

721 heating for $10 \mathrm{~min}$ at $99^{\circ} \mathrm{C}$.

723 In Fig. 6a, lysis buffer composition was 25 mM HEPES pH 7.4, 1 mM DTT, 0.2 \% NP-40, 0.5 mM

724 Mg-ATP, $1 \mathrm{mM}$ EGTA, $10 \%$ glycerol, $2 \mathrm{mM}$ magnesium acetate, $50 \mathrm{mM}$ potassium acetate, 
725 supplemented with complete EDTA-free protease inhibitor capsule. Lysates were incubated

726 with $30 \mu \mathrm{l}$ magnetic-GFP trap (homemade, detailed below) at $4{ }^{\circ} \mathrm{C}$, $2 \mathrm{~h}$ with gentle rotation.

727 Following incubation, cells were washed with lysis buffer without complete EDTA-free

728 protease inhibitor capsule.

729

\section{Real-time qRT-PCR}

731 To determine knock-down efficiency, we used quantitative reverse transcription PCR (qRT732 PCR). Briefly, total RNA was extracted from cells treated with non-targeting siRNA (siNT) or 733 siRNA targeting RUFY3 or RUFY4, using the RNeasy Mini Kit (Cat\# 74106, Qiagen) according 734 to manufacturer's instructions. Complementary DNA was generated by reverse transcription 735 using the Superscript VILO cDNA Synthesis Kit (11754050, Thermo Fisher), using 50 ng of the 736 extracted mRNA as template. The cDNA was diluted 1:100 in PCR-grade water and used as 737 template for qPCR with TaqMan ${ }^{\circledR}$ Gene Expression assays (Thermo Fisher) targeting either 738 human RUFY3 (Cat\# 4448892, Hs01127885_m1) , RUFY4 (Cat\# 4448892, Hs01651015_m1) or the 739 housekeeping gene ACTB (Cat\# 4448489, Hs01060665_g1) in the TaqMan Fast Advanced Master

740 Mix (Cat\# 4444557, Thermo Fisher). qPCR was performed on the AriaMx Real-Time PCR

741 system using the AriaMx software version 1.3 (Agilent Technologies)

\section{Preparation of rat hippocampal neurons}

744 Rat hippocampal neurons were isolated as previously described (Farias et al., 2016). Briefly, E18 rat embryos were harvested and euthanized. The brains were isolated in Hank's medium, and hippocampi were dissociated mechanically with a narrow-mouth glass pipette followed by trypsinization with $0.25 \%$ trypsin (Cat\# 1509046, Gibco) for $15 \mathrm{~min}$ at $37 \mathrm{C}$. Cells were plated on 18-mm microscopic glass coverslips coated with polylysine (Cat\# 11243217001, Roche) and laminin (5 $\mathrm{gg} / \mathrm{mL}$ ) (Cat\# P2636, Millipore-Sigma) in DMEM with $4.5 \mathrm{~g} / \mathrm{L}$ glucose, $25 \mathrm{mM}$ HEPES, 10\% heat-inactivated horse serum (Cat\# 26050-088, Gibco), $100 \mathrm{U} / \mathrm{mL}$ penicillin and $100 \mu \mathrm{g} / \mathrm{mL}$ streptomycin. Three hours post plating, the medium was replaced with Neurobasal medium (Cat\# 21103-049, Gibco), supplemented with 1X B27 (Cat\# 17504044, Thermo

753 Scientific), Glutamax (Cat\# 35050-61, Life Technologies), and $100 \mathrm{U} / \mathrm{mL}$ penicillin-streptomycin

754 (Cat\# 15140148, Gibco) and placed at $37^{\circ} \mathrm{C}$ and $5 \% \mathrm{CO}_{2}$.

\section{Transfection and immunofluorescence microscopy of neurons}

757 Rat hippocampal neurons were transfected at Day-in-vitro 4 (DIV4) using 1.2 $\mu$ L Lipofectamine 7582000 mixed in $200 \mu \mathrm{L}$ of Opti-MEM with 1-2 $\mu$ g plasmid DNA per 18-mm cover glass with 800 
$759 \mu \mathrm{L}$ Neurobasal medium for $1 \mathrm{~h}$ at 37 'C. After $1 \mathrm{~h}$, Lipofectamine 2000 was washed with

760 Neurobasal medium and the cells were kept in fresh, complete Neurobasal medium for $24 \mathrm{~h}$.

761 For immunofluorescence microscopy, neurons were fixed with $4 \%$ PFA in PBS supplemented

762 with $4 \%$ sucrose, $0.1 \mathrm{mM} \mathrm{CaCl}_{2}$ and $1 \mathrm{mM} \mathrm{MgCl}_{2}$ (PBS-CM) for $20 \mathrm{~min}$. Cells were

763 permeabilized with $0.2 \% \mathrm{v} / \mathrm{v}$ Triton X-100 for $15 \mathrm{~min}$ at room temperature. After that, cells

764 were incubated with $0.2 \%$ gelatin in PBS-CM for $30 \mathrm{~min}$. Primary and secondary antibodies

765 were prepared in blocking solution and incubated for $30 \mathrm{~min}$ each at $37{ }^{\circ} \mathrm{C}$. Cells were mounted

766 with Fluoromount G (Electron Microscopy Sciences). Images were taken in a Zeiss LSM 780

767 confocal microscope using a Plan Apochromat 63x objective (N.A. 1.40).

\section{Live imaging of neurons}

770 To analyze lysosome movement, neurons were co-transfected at DIV4 with plasmids encoding

771 RUFY3-GFP or RUFY4-GFP along with LAMP1-RFP and imaged $24 \mathrm{~h}$ post-transfection. In live

772 neurons, axons were identified by labeling with CF640R (Biotium)-conjugated antibody to the

773 axon initial segment (AIS) protein neurofascin (Farias et al 2016). Videos were recorded at 200

774 milliseconds for individual channels without any delay for a total of $5 \mathrm{~min}$. Live-cell imaging

775 was performed on a spinning-disk Eclipse Ti Microscope System (Nikon) equipped with a

776 humidified environmental chamber maintained at $37{ }^{\circ} \mathrm{C}$ and $5 \% \mathrm{CO}_{2}$. Images were acquired

777 with NIS-Elements AR microscope imaging software using a high-speed EMCCD camera (iXon

778 Life 897, Andor). Axonal kymographs were generated using Fiji software with segmented line

779 tool of one-pixel thickness along a segment of the axon just distal to the AIS, followed by stack

780 re-slicing projection.

782 Expression and purification of recombinant proteins

783 BL21-CodonPlus (DE3) RP E. coli cells (Cat\# 230255, Agilent Technologies) expressing target

784 proteins were grown in $1 \mathrm{~L}$ Terrific Broth supplemented with $34 \mu \mathrm{g} / \mathrm{mL}$ chloramphenicol (C-

785 6378, Millipore-Sigma) and $100 \mu \mathrm{g} / \mathrm{mL}$ ampicillin (Cat\# A1066, Millipore-Sigma) for GST-

786 plasmids or $30 \mu \mathrm{g} / \mathrm{mL}$ kanamycin (Cat\# K1377, Millipore-Sigma) for 6His-sfGFP plasmids.

787 Cultures were grown for $6-8 \mathrm{~h}$ at $37^{\circ} \mathrm{C}$, with $200 \mathrm{rpm}$ rotation, induced with $1 \mathrm{mM}$ IPTG

788 (isopropyl $\beta$-D-1-thiogalactopyranoside) (Cat\# I2481, GoldBio) and incubated overnight at 16-18

$789{ }^{\circ} \mathrm{C}, 200 \mathrm{rpm}$. Bacterial cultures were pelleted by centrifugation for $20 \mathrm{~min}$ at $4{ }^{\circ} \mathrm{C}, 4,000 \mathrm{rpm}$.

790 and pellets were resuspended in lysis buffer supplemented with lysozyme (Cat\# VWRV0663,

791 VWR), DNase I (Cat\# LS002139, Worthington Biochemical Corporation) and complete EDTA-

792 free protease inhibitor capsules (Cat\# 1836170, Roche) (specific buffers used are listed below for 
793 each protein purified in this study). Following sonication and centrifugation for 30-45 min at

$7944^{\circ} \mathrm{C}, 16,000 \mathrm{rpm}$, cleared lysates were incubated with glutathione-Sepharose 4B (Cat\# 17-0756-

795 05, Cytvia) (for GST- tagged proteins) or cOmplete-His-Tag Purification Resin (Cat\#

7965893682001 , Roche) (for 6His-sfGFP-tagged proteins) for $1-2 \mathrm{~h}$ at $4{ }^{\circ} \mathrm{C}$, with gentle end-to-end

797 rotation.

798

799 For purification of GST-ARL8B-Q75L and -T34N, lysis buffer was $50 \mathrm{mM}$ Tris-HCl pH 8.0, 150

$800 \mathrm{mM} \mathrm{NaCl}, 8 \mathrm{mM} \mathrm{MgCl} 2,5 \%$ glycerol and $5 \mathrm{mM} \beta$-Mercaptoethanol (Cat\# M6250, Millipore-

801 Sigma) supplemented with 100 MM GDP (Cat\# G7127, Millipore-Sigma) (for GST-ARL8B-T34N)

802 or $100 \mu \mathrm{M} \mathrm{GTP} \gamma \mathrm{S}$ (Cat\# G8634, Millipore-Sigma) (for GST-ARL8B-Q75L). Bound glutathione-

803 Sepharose was washed in buffer containing $50 \mathrm{mM}$ Tris- $\mathrm{HCl} \mathrm{pH}$ 8.0, $150 \mathrm{mM} \mathrm{NaCl}, 8 \mathrm{mM}$

$804 \mathrm{MgCl}_{2}, 5 \%$ glycerol and $5 \mathrm{mM} \beta$-Mercaptoethanol (Cat\# M6250, Millipore-Sigma) supplemented

805 with $100 \mu \mathrm{M}$ GDP (G7127, Millipore-Sigma) (for GST-ARL8B-T34N) or $100 \mu \mathrm{M}$ GTP $\gamma S$ (Cat\#

806 G8634, Millipore-Sigma) (for GST-ARL8B-Q75L).

807

808 GST-DLIC1, and GST-DLIC-CT were expressed in BL21(DE3) (Cat\# C2527I, New England

809 Biolabs) and lysis buffer was $50 \mathrm{mM}$ Tris- $\mathrm{HCl} \mathrm{pH}$ 8.0, $150 \mathrm{mM} \mathrm{NaCl}, 10 \%$ glycerol, and $1 \mathrm{mM}$

810 DTT (Cat\# DTT-RO, Roche). Following binding to glutathione Sepharose and washes, bound

811 protein was further eluted from the glutathioneSepharose with elution buffer containing $50 \mathrm{mM}$

812 Tris-HCl pH 8.0 and $10 \mathrm{mM} \mathrm{L-glutathione.} \mathrm{Eluant} \mathrm{was} \mathrm{further} \mathrm{purified} \mathrm{on} \mathrm{HiLoad} \mathrm{16/600}$

813 Superdex 200 (Cat\# 28-9893-35, Cytvia) in buffer containing $10 \mathrm{mM}$ Tris-HCl pH 7.0, $50 \mathrm{mM}$

$814 \mathrm{NaCl}, 2 \mathrm{mM} \mathrm{MgCl}$ and 2mM Tris(2-carboxyethyl)phosphine hydrochloride (TCEP) (Cat\#

815 C4706, Millipore-Sigma). Peak fractions were pooled together, aliquoted, flash-frozen in liquid

816 nitrogen and stored at $-80^{\circ} \mathrm{C}$

817

818 For 6His-sfGFP-RUFY3, 6His-sfGFP-BICD2 and 6His-sfGFP-GFP purification, lysis buffer was

$81950 \mathrm{mM}$ Tris-HCl pH 8.0, $300 \mathrm{mM} \mathrm{NaCl}, 5$ \% glycerol and 1 mM DTT (Cat\# 10708984001,

820 Millipore-Sigma). Bound proteins on cOmplete-His-Tag Purification Resin (Cat\# 5893682001,

821 Roche) were washed in buffer containing $50 \mathrm{mM}$ Tris- $\mathrm{HCl} \mathrm{pH}$ 8.0, $300 \mathrm{mM} \mathrm{NaCl}, 5 \%$ glycerol

822 and eluted in buffer composed of $50 \mathrm{mM}$ Tris- $\mathrm{HCl} \mathrm{pH}$ 8.0, $300 \mathrm{mM} \mathrm{NaCl}, 5 \%$ glycerol with 1

$823 \mathrm{mM}$ DTT and $90 \mathrm{mM}$ imidazole. Proteins were further purified on Superose 6 increase 10/300

824 column (Cat\# 29-0915-96, Cytvia) in $50 \mathrm{mM}$ Tris-HCl pH 8.0, $300 \mathrm{mM} \mathrm{NaCl}, 5$ \% glycerol and 1

825 mM DTT. Peak fractions were pooled together, aliquoted, flash-frozen at liquid nitrogen and

826 stored at $-80^{\circ} \mathrm{C}$.

827 
828

829

830

831

832

833

834

835

836

837

838

839

840

841

842

843

844

845

846

847

848

849

850

851

852

853

854

855

856

857

858

859

860

861

862

\section{Preparation of GFP-nanobody conjugated agarose}

Homemade GFP-Trap beads were generated by first purifying the GFP nanobody, then coupling it to N-hydroxysuccinimide (NHS) beads (Cat\# GE28-9513-80, Millipore-Sigma).

E.coli BL21 (DE3) were transformed with pOPINE-GFPnanobody plasmid, (Kubala et al., 2010) (a gift from Brett Collins, Addgene \#49172). GFP nanobody was expressed as described above with buffer composed of $50 \mathrm{mM}$ Tris- $\mathrm{HCl}, 300 \mathrm{mM} \mathrm{NaCl}, 5 \%$ glycerol and $1 \mathrm{mM}$ DTT that was supplemented with DNAseI, lysozyme and complete EDTA free tablet. cOmplete His-Tag purification resin was prepared by washing $5 \mathrm{~mL}$ of resin with cold PBS. The cleared lysate was incubated in batch mode with the cOmplete His-Tag purification resin for $30 \min$ at $4{ }^{\circ} \mathrm{C}$, with end-to-end rotation. The lysate was removed, the resin washed with $600 \mathrm{~mL}$ cold PBS and the proteins eluted in PBS supplemented with $500 \mathrm{mM}$ imidazole. The elution was conducted 4 times for a total elution volume of $20 \mathrm{~mL}$. Eluant was dialyzed in $4 \mathrm{~L}$ PBS supplemented with $150 \mathrm{mM} \mathrm{NaCl}$ overnight at $4{ }^{\circ} \mathrm{C}$. The nanobody was additionally purified by gel filtration on a Superdex 200 increase 300 / 10 column (Cat\# 28-9909-44, Cytvia) in 25 mM HEPES pH 7.4, 150 $\mathrm{mM} \mathrm{NaCl}$. Peak fractions were pooled and purified nanobody at $1.8 \mathrm{mg} / \mathrm{ml}$ concentration was aliquoted, flash frozen and stored at $-80^{\circ} \mathrm{C}$ while $1 \mathrm{mg}$ was used to prepare GFP-Trap beads.

Coupling of the nanobody to NHS Mag Sepharose (Cat\# GE28-9513-80, Millipore-Sigma) was conducted according to supplier's specifications. Briefly, one $500 \mu \mathrm{L}$ tube of NHS Mag Sepharose was placed on a magnetic rack and the storage solution was removed. The beads were equilibrated by resuspending them in $500 \mu \mathrm{L}$ ice-cold $1 \mathrm{M} \mathrm{HCl}$ and removing the liquid. The $1 \mathrm{mg}$ of purified nanobody, diluted to $1 \mathrm{~mL}$ in $0.2 \mathrm{M} \mathrm{NaHCO}_{3}, 0.5 \mathrm{M} \mathrm{NaCl}, \mathrm{pH} 8.3$, was added to the beads and allowed to mix end-over-end at room temperature for $20 \mathrm{~min}$. The nanobody solution was then removed and residual active groups were blocked by sequential washes in $50 \mathrm{mM}$ Tris-HCl, $1 \mathrm{M} \mathrm{NaCl}, \mathrm{pH} 8$ (Buffer A) and $50 \mathrm{mM}$ glycine- $\mathrm{HCl}, 1 \mathrm{M} \mathrm{NaCl}, \mathrm{pH}$ 3.0 (Buffer B). The washes were as follows: $500 \mu \mathrm{L}$ Buffer A, $500 \mu \mathrm{L}$ Buffer B, $500 \mu \mathrm{L}$ Buffer A, mixed end-over-end at room temperature for $15 \mathrm{~min}$. The buffer was removed. The beads were sequentially washed in $500 \mu \mathrm{L}$ Buffer B, $500 \mu \mathrm{L}$ Buffer A and $500 \mu \mathrm{L}$ Buffer B. The beads were resuspended in $500 \mu \mathrm{L}$ of $50 \mathrm{mM}$ Tris- $\mathrm{HCl} \mathrm{pH} 7.4$ containing $20 \%$ ethanol and stored at $4^{\circ} \mathrm{C}$.

\section{Pull downs}

HEK293T cells expressing RUFYs or RUFY-FLAG plasmids were scraped from 10-cm plates and washed 3 times in $1 \mathrm{~mL}$ cold PBS followed by centrifugation for $5 \mathrm{~min}$ at $4{ }^{\circ} \mathrm{C}, 500 \times \mathrm{g}$. Pellets were resuspended in $1 \mathrm{~mL}$ buffer containing $25 \mathrm{mM}$ Tris- $\mathrm{HCl} \mathrm{pH}$ 7.4, $150 \mathrm{mM} \mathrm{NaCl}, 1 \mathrm{mM}$ EDTA, $1 \%$ NP-40 (Cat\# 011332473001, Roche) and $5 \%$ glycerol, supplemented with complete 
863 EDTA-free protease inhibitor capsule (Cat\# 1836170, Roche) and 500 $\mu$ M GDP (Cat\# G7127,

864 Millipore-Sigma) (for GST-ARL8B-T34N) or $500 \mu \mathrm{M}$ GTP $\gamma S$ (Cat\# G8634, Millipore-Sigma) (for

865 GST-ARL8B-Q75L), 1 mM DL-Dithiothreitol (DTT) (Cat\# 10708984001, Millipore-Sigma) and 8

$866 \mathrm{mM} \mathrm{MgCl}_{2}$, and incubated for $30 \mathrm{~min}$ at $4{ }^{\circ} \mathrm{C}$ with gentle rotation. Lysates were entrifuged for 10

867 min at $4{ }^{\circ} \mathrm{C}, 17,000 \times \mathrm{g}$ and incubated with $20 \mu$ glutathione-Sepharose loaded with GST-

868 ARL8B-Q75L or GST-ARL8B-T34N for $1 \mathrm{~h}$ at $4{ }^{\circ} \mathrm{C}$ with gentle rotation (preparation of GST-

869 ARL8B-Q75L and -T34N is described above). Bound material was separated by centrifugation

870 for $5 \mathrm{~min}$ at $4{ }^{\circ} \mathrm{C}, 500 \times \mathrm{g}$, and washed 3 times with $1 \mathrm{~mL}$ buffer containing $50 \mathrm{mM}$ Tris- $\mathrm{HCl} \mathrm{pH}$

$8718.0,150 \mathrm{mM} \mathrm{NaCl}, 8 \mathrm{mM} \mathrm{MgCl}$, $5 \%$ glycerol and $5 \mathrm{mM} \beta$-Mercaptoethanol (Cat\# M6250,

872 Millipore-Sigma) supplemented with $100 \mu$ M GDP (Cat\# G7127, Millipore-Sigma) (for GST-

873 ARL8B-T34N) or GTPүS (G8634, Millipore-Sigma) (for GST-ARL8B-Q75L) (Cat\# 10708984001,

874 Millipore-Sigma). Samples were eluted with Laemmli sample buffer for $10 \mathrm{~min}$ at $99^{\circ} \mathrm{C}$.

For the pull down with GST-DLIC and GST-DLIC-CT, $20 \mu \mathrm{g}$ protein were incubated with $20 \mu \mathrm{l}$ glutathione-Sepharose. Loaded GST-beads were incubated with $5 \mu \mathrm{g}$ purified 6His-sfGFPRUFY3 for $1 \mathrm{~h}$ at $4{ }^{\circ} \mathrm{C}$ with gentle rotation.

\section{Endogenous dynein pulldown}

881 We used a published protocol (Kesisova et al., 2021; McKenney et al., 2014) with modifications. 882 HEK293T cells from fifteen 15-cm plates were scraped and washed 3 times in cold PBS for 5 min 883 at $4{ }^{\circ} \mathrm{C}, 500 \times g$ centrifugations between washes. Cells were lysed in $15 \mathrm{ml}$ buffer composed of 25 884 mM HEPES pH 7.4, 5 mM DTT, 0.2 \% NP40, 1 mM Mg-ATP, 1 mM EGTA, $10 \%$ glycerol, 2 mM magnesium acetate, $50 \mathrm{mM}$ potassium acetate supplemented with complete EDTA-free protease inhibitor capsule for $1 \mathrm{~h}$ at $4^{\circ} \mathrm{C}$ with gentle rotation. The supernatant was separated by centrifugation for $30 \mathrm{~min}$ at $4^{\circ} \mathrm{C}$ and 120,000 $\mathrm{g}$ (TLA45). $3.5 \mathrm{~mL}$ of the cleared HEK293T lysate was mixed with $100 \mu$ l Strep-Tactin Sepharose resin (Cat\# 2-1201-010, IBA) and also $40 \mu \mathrm{g}$ purified 6His-sfGFP-RUFY3, 6His-sfGFP-BICD2 and 6His-sfGFP and incubated over-night at 4 ${ }^{\circ} \mathrm{C}$ with gentle rotation. Following incubation, beads were washed 5 times in $2 \mathrm{~mL}$ buffer for 3 $\min$ at $4{ }^{\circ} \mathrm{C}$ and $500 \times g$ spins between washes. Samples were further eluted with $50 \mu \mathrm{l}$ X Laemmli sample buffer, $10 \mathrm{~min}$ at $99^{\circ} \mathrm{C}$.

\section{Statistical calculations}

895 All statistical tests were performed on $n=3$ independent experiments, except in Fig. 8c where 4089650 cells were analyzed per condition in one experiment. Data are presented as superplots (Lord 
897 et al., 2020). Individual data points from each experiment are color coded and correspond to the 898 big circles representing the average for each experiment. Error bars show standard deviation. 899 We used One-way ANOVA when multiple groups were compared and unpaired Student's t900 test when two groups were compared. Data in Figs. 4c,e,h,I were normalized to GFP. This was 901 done to account for experiment-to-experiment variability (the trends within each experiment 902 was always consistent).

903 
904

905

906

907

908

909

910

911

912

913

914

915

916

\section{References}

Adnan, G., Rubikaite, A., Khan, M., Reber, M., Suetterlin, P., Hindges, R., and Drescher, U. (2020). The GTPase Arl8B Plays a Principle Role in the Positioning of Interstitial Axon Branches by Spatially Controlling Autophagosome and Lysosome Location. J Neurosci 40, 8103-8118.

Antonny, B., Beraud-Dufour, S., Chardin, P., and Chabre, M. (1997). N-terminal hydrophobic residues of the G-protein ADP-ribosylation factor-1 insert into membrane phospholipids upon GDP to GTP exchange. Biochemistry 36, 4675-4684.

Bagshaw, R.D., Callahan, J.W., and Mahuran, D.J. (2006). The Arf-family protein, Arl8b, is involved in the spatial distribution of lysosomes. Biochem Biophys Res Commun 344, 1186-1191.

Ballabio, A., and Bonifacino, J.S. (2020). Lysosomes as dynamic regulators of cell and organismal homeostasis. Nat Rev Mol Cell Biol 21, 101-118.

Bonifacino, J.S., and Neefjes, J. (2017). Moving and positioning the endolysosomal system. Curr Opin Cell Biol 47, 1-8.

Bordat, A., Houvenaghel, M.C., and German-Retana, S. (2015). Gibson assembly: an easy way to clone potyviral full-length infectious cDNA clones expressing an ectopic VPg. Virol J 12, 89.

Boucrot, E., Henry, T., Borg, J.P., Gorvel, J.P., and Meresse, S. (2005). The intracellular fate of Salmonella depends on the recruitment of kinesin. Science 308, 1174-1178.

Britton, S., Coates, J., and Jackson, S.P. (2013). A new method for high-resolution imaging of Ku foci to decipher mechanisms of DNA double-strand break repair. J Cell Biol 202, 579-595.

Cai, Q., Lu, L., Tian, J.H., Zhu, Y.B., Qiao, H., and Sheng, Z.H. (2010). Snapin-regulated late endosomal transport is critical for efficient autophagy-lysosomal function in neurons. Neuron 68, 73-86.

Cason, S.E., Carman, P., Van Duyne, C., Goldsmith, J., Dominguez, R., and Holzbaur, E.L.F. (2020). Sequential dynein effectors regulate axonal autophagosome motility in a maturation-dependent pathway. bioRxiv, https: / / doi.org/10.1101/2020.11.01.363333 
933 Char, R., and Pierre, P. (2020). The RUFYs, a Family of Effector Proteins Involved in

934 Intracellular Trafficking and Cytoskeleton Dynamics. Front Cell Dev Biol 8, 779.

935 Cormont, M., Mari, M., Galmiche, A., Hofman, P., and Le Marchand-Brustel, Y. (2001). A FYVE-

936 finger-containing protein, Rabip4, is a Rab4 effector involved in early endosomal traffic.

937 Proc Natl Acad Sci U S A 98, 1637-1642.

938 De Pace, R., Britt, D.J., Mercurio, J., Foster, A.M., Djavaherian, L., Hoffmann, V., Abebe, D., and 939 Bonifacino, J.S. (2020). Synaptic Vesicle Precursors and Lysosomes Are Transported by 940 Different Mechanisms in the Axon of Mammalian Neurons. Cell Rep 31, 107775.

941 Drerup, C.M., and Nechiporuk, A.V. (2013). JNK-interacting protein 3 mediates the retrograde 942 transport of activated c-Jun N-terminal kinase and lysosomes. PLoS Genet 9, e1003303.

943 Dykes, S.S., Gray, A.L., Coleman, D.T., Saxena, M., Stephens, C.A., Carroll, J.L., Pruitt, K., and 944 Cardelli, J.A. (2016). The Arf-like GTPase Arl8b is essential for three-dimensional 945 invasive growth of prostate cancer in vitro and xenograft formation and growth in vivo. $946 \quad$ Oncotarget 7, 31037-31052.

947 Farfel-Becker, T., Roney, J.C., Cheng, X.T., Li, S., Cuddy, S.R., and Sheng, Z.H. (2019). Neuronal 948 Soma-Derived Degradative Lysosomes Are Continuously Delivered to Distal Axons to 949 Maintain Local Degradation Capacity. Cell Rep 28, 51-64 e54.

950 Farias, G.G., Britt, D.J., and Bonifacino, J.S. (2016). Imaging the Polarized Sorting of Proteins 951 from the Golgi Complex in Live Neurons. Methods Mol Biol 1496, 13-30.

952 Farias, G.G., Guardia, C.M., De Pace, R., Britt, D.J., and Bonifacino, J.S. (2017). BORC/ kinesin-1 953 ensemble drives polarized transport of lysosomes into the axon. Proc Natl Acad Sci U S 954 A 114, E2955-E2964.

955 Fermie, J., Liv, N., Ten Brink, C., van Donselaar, E.G., Muller, W.H., Schieber, N.L., Schwab, Y., 956 Gerritsen, H.C., and Klumperman, J. (2018). Single organelle dynamics linked to 3D 957 structure by correlative live-cell imaging and 3D electron microscopy. Traffic 19, 354958369.

959 French, A.P., Mills, S., Swarup, R., Bennett, M.J., and Pridmore, T.P. (2008). Colocalization of 960 fluorescent markers in confocal microscope images of plant cells. Nat Protoc 3, 619-628.

961 Fukuda, M., and Itoh, T. (2008). Direct link between Atg protein and small GTPase Rab: Atg16L 962 functions as a potential Rab33 effector in mammals. Autophagy 4, 824-826. 
963 Fukuda, M., Kobayashi, H., Ishibashi, K., and Ohbayashi, N. (2011). Genome-wide investigation of the Rab binding activity of RUN domains: development of a novel tool that specifically traps GTP-Rab35. Cell Struct Funct 36, 155-170.

Garg, S., Sharma, M., Ung, C., Tuli, A., Barral, D.C., Hava, D.L., Veerapen, N., Besra, G.S., Hacohen, N., and Brenner, M.B. (2011). Lysosomal trafficking, antigen presentation, and microbial killing are controlled by the Arf-like GTPase Arl8b. Immunity 35, 182-193.

Ghosh, S., Dellibovi-Ragheb, T.A., Kerviel, A., Pak, E., Qiu, Q., Fisher, M., Takvorian, P.M., Bleck, C., Hsu, V.W., Fehr, A.R., et al. (2020). beta-Coronaviruses Use Lysosomes for

Gillingham, A.K., Bertram, J., Begum, F., and Munro, S. (2019). In vivo identification of GTPase interactors by mitochondrial relocalization and proximity biotinylation. Elife 8 .

Gosney, J.A., Wilkey, D.W., Merchant, M.L., and Ceresa, B.P. (2018). Proteomics reveals novel protein associations with early endosomes in an epidermal growth factor-dependent manner. J Biol Chem 293, 5895-5908.

Guardia, C.M., De Pace, R., Sen, A., Saric, A., Jarnik, M., Kolin, D.A., Kunwar, A., and Bonifacino, J.S. (2019). Reversible association with motor proteins (RAMP): A

Guardia, C.M., Farias, G.G., Jia, R., Pu, J., and Bonifacino, J.S. (2016). BORC Functions Upstream of Kinesins 1 and 3 to Coordinate Regional Movement of Lysosomes along Different Microtubule Tracks. Cell Rep 17, 1950-1961.

Hertz, N.T., Adams, E.L., Weber, R.A., Shen, R.J., O'Rourke, M.K., Simon, D.J., Zebroski, H., Olsen, O., Morgan, C.W., Mileur, T.R., et al. (2019). Neuronally Enriched RUFY3 Is vesiculation and lysosome dispersion in cells lacking cytoplasmic dynein. J Cell Biol 141, 51-59. Required for Caspase-Mediated Axon Degeneration. Neuron 103, 412-422 e414. 
992 Heuser, J. (1989). Changes in lysosome shape and distribution correlated with changes in 993 cytoplasmic pH. J Cell Biol 108, 855-864.

994 Hofmann, I., and Munro, S. (2006). An N-terminally acetylated Arf-like GTPase is localised to 995 lysosomes and affects their motility. J Cell Sci 119, 1494-1503.

996 Hollenbeck, P.J., and Swanson, J.A. (1990). Radial extension of macrophage tubular lysosomes 997 supported by kinesin. Nature 346, 864-866.

998 Honda, A., Usui, H., Sakimura, K., and Igarashi, M. (2017). Rufy3 is an adapter protein for small 999 GTPases that activates a Rac guanine nucleotide exchange factor to control neuronal $1000 \quad$ polarity. J Biol Chem 292, 20936-20946.

1001 Jackson, C.L., and Bouvet, S. (2014). Arfs at a glance. J Cell Sci 127, 4103-4109.

1002 Jia, R., and Bonifacino, J.S. (2019). Lysosome Positioning Influences mTORC2 and AKT 1003 Signaling. Mol Cell 75, 26-38 e23.

1004 Jia, R., Guardia, C.M., Pu, J., Chen, Y., and Bonifacino, J.S. (2017). BORC coordinates encounter 1005 and fusion of lysosomes with autophagosomes. Autophagy 13, 1648-1663.

1006 Johnson, D.E., Ostrowski, P., Jaumouille, V., and Grinstein, S. (2016). The position of lysosomes 1007 within the cell determines their luminal pH. J Cell Biol 212, 677-692.

1008 Jongsma, M.L., Bakker, J., Cabukusta, B., Liv, N., van Elsland, D., Fermie, J., Akkermans, J.L., 1009 Kuijl, C., van der Zanden, S.Y., Janssen, L., et al. (2020). SKIP-HOPS recruits TBC1D15 for 1010 a Rab7-to-Arl8b identity switch to control late endosome transport. EMBO J 39, e102301.

1011 Jongsma, M.L., Berlin, I., Wijdeven, R.H., Janssen, L., Janssen, G.M., Garstka, M.A., Janssen, H., 1012 Mensink, M., van Veelen, P.A., Spaapen, R.M., et al. (2016). An ER-Associated Pathway 1013 Defines Endosomal Architecture for Controlled Cargo Transport. Cell 166, 152-166.

1014 Jordens, I., Fernandez-Borja, M., Marsman, M., Dusseljee, S., Janssen, L., Calafat, J., Janssen, H., 1015 Wubbolts, R., and Neefjes, J. (2001). The Rab7 effector protein RILP controls lysosomal 1016 transport by inducing the recruitment of dynein-dynactin motors. Curr Biol 11, 168010171685.

1018 Kanaji, S., Iwahashi, J., Kida, Y., Sakaguchi, M., and Mihara, K. (2000). Characterization of the 1019 signal that directs Tom20 to the mitochondrial outer membrane. J Cell Biol 151, 277-288. 
1020 Kapitein, L.C., Schlager, M.A., van der Zwan, W.A., Wulf, P.S., Keijzer, N., and Hoogenraad, C.C. (2010). Probing intracellular motor protein activity using an inducible cargo trafficking assay. Biophys J 99, 2143-2152.

1023 Keren-Kaplan, T., and Bonifacino, J.S. (2021). ARL8 Relieves SKIP Autoinhibition to Enable Coupling of Lysosomes to Kinesin-1. Curr Biol 31, 540-554 e545.

1025 Kesisova, I.A., Robinson, B.P., and Spiliotis, E.T. (2021). A septin GTPase scaffold of dyneindynactin motors triggers retrograde lysosome transport. J Cell Biol 220.

1027 1028 1029

1030

1031

1032

1033

1034

1035

1036

1037

1038

1039

1040

1041

1042

1043

1044

1045

1046

1047

Khatter, D., Raina, V.B., Dwivedi, D., Sindhwani, A., Bahl, S., and Sharma, M. (2015a). The small GTPase Arl8b regulates assembly of the mammalian HOPS complex on lysosomes. J Cell Sci 128, 1746-1761.

Khatter, D., Sindhwani, A., and Sharma, M. (2015b). Arf-like GTPase Arl8: Moving from the periphery to the center of lysosomal biology. Cell Logist 5, e1086501.

Kim, D.I., Jensen, S.C., Noble, K.A., Kc, B., Roux, K.H., Motamedchaboki, K., and Roux, K.J. (2016). An improved smaller biotin ligase for BioID proximity labeling. Mol Biol Cell 27, 1188-1196.

Klassen, M.P., Wu, Y.E., Maeder, C.I., Nakae, I., Cueva, J.G., Lehrman, E.K., Tada, M., GengyoAndo, K., Wang, G.J., Goodman, M., et al. (2010). An Arf-like small G protein, ARL-8, promotes the axonal transport of presynaptic cargoes by suppressing vesicle aggregation. Neuron 66, 710-723.

Korolchuk, V.I., Saiki, S., Lichtenberg, M., Siddiqi, F.H., Roberts, E.A., Imarisio, S., Jahreiss, L., Sarkar, S., Futter, M., Menzies, F.M., et al. (2011). Lysosomal positioning coordinates cellular nutrient responses. Nat Cell Biol 13, 453-460.

Kubala, M.H., Kovtun, O., Alexandrov, K., and Collins, B.M. (2010). Structural and thermodynamic analysis of the GFP:GFP-nanobody complex. Protein Sci 19, 2389-2401.

Lassen, K.G., McKenzie, C.I., Mari, M., Murano, T., Begun, J., Baxt, L.A., Goel, G., Villablanca, E.J., Kuo, S.Y., Huang, H., et al. (2016). Genetic Coding Variant in GPR65 Alters Lysosomal $\mathrm{pH}$ and Links Lysosomal Dysfunction with Colitis Risk. Immunity 44, 13921405. 
1048 Lee, I.G., Olenick, M.A., Boczkowska, M., Franzini-Armstrong, C., Holzbaur, E.L.F., and

1049

1050

1051

1052

1053

1054

1055

1056

1057

1058

1059

1060

1061

1062

1063

1064

1065

1066

1067

1068

1069

1070

1071

1072

1073

1074

1075

1076

1077

Dominguez, R. (2018). A conserved interaction of the dynein light intermediate chain with dynein-dynactin effectors necessary for processivity. Nat Commun 9, 986.

Lee, S., Sato, Y., and Nixon, R.A. (2011). Lysosomal proteolysis inhibition selectively disrupts axonal transport of degradative organelles and causes an Alzheimer's-like axonal dystrophy. J Neurosci 31, 7817-7830.

Levin-Konigsberg, R., Montano-Rendon, F., Keren-Kaplan, T., Li, R., Ego, B., Mylvaganam, S., DiCiccio, J.E., Trimble, W.S., Bassik, M.C., Bonifacino, J.S., et al. (2019). Phagolysosome resolution requires contacts with the endoplasmic reticulum and phosphatidylinositol-4phosphate signalling. Nat Cell Biol 21, 1234-1247.

Li, X., Rydzewski, N., Hider, A., Zhang, X., Yang, J., Wang, W., Gao, Q., Cheng, X., and Xu, H. (2016). A molecular mechanism to regulate lysosome motility for lysosome positioning and tubulation. Nat Cell Biol 18, 404-417.

Liu, Y., Kahn, R.A., and Prestegard, J.H. (2009). Structure and membrane interaction of myristoylated ARF1. Structure 17, 79-87.

Lord, S.J., Velle, K.B., Mullins, R.D., and Fritz-Laylin, L.K. (2020). SuperPlots: Communicating reproducibility and variability in cell biology. J Cell Biol 219.

Lund, V.K., Lycas, M.D., Schack, A., Andersen, R.C., Gether, U., and Kjaerulff, O. (2021). Rab2 drives axonal transport of dense core vesicles and lysosomal organelles. Cell Rep 35, 108973.

Marwaha, R., Arya, S.B., Jagga, D., Kaur, H., Tuli, A., and Sharma, M. (2017). The Rab7 effector PLEKHM1 binds Arl8b to promote cargo traffic to lysosomes. J Cell Biol 216, 1051-1070.

McKenney, R.J., Huynh, W., Tanenbaum, M.E., Bhabha, G., and Vale, R.D. (2014). Activation of cytoplasmic dynein motility by dynactin-cargo adapter complexes. Science 345, 337-341.

Men, W., Li, W., Li, Y., Zhao, J., Qu, X., Li, P., and Gong, S. (2019). RUFY3 Predicts Poor Prognosis and Promotes Metastasis through Epithelial-mesenchymal Transition in Lung Adenocarcinoma. J Cancer 10, 6278-6285.

Mori, T., Wada, T., Suzuki, T., Kubota, Y., and Inagaki, N. (2007). Singar1, a novel RUN domaincontaining protein, suppresses formation of surplus axons for neuronal polarity. J Biol Chem 282, 19884-19893. 
1078 Mrakovic, A., Kay, J.G., Furuya, W., Brumell, J.H., and Botelho, R.J. (2012). Rab7 and Arl8 GTPases are necessary for lysosome tubulation in macrophages. Traffic 13, 1667-1679.

1080 Nag, S., Rani, S., Mahanty, S., Bissig, C., Arora, P., Azevedo, C., Saiardi, A., van der Sluijs, P., Delevoye, C., van Niel, G., et al. (2018). Rab4A organizes endosomal domains for sorting

Niwa, S., Lipton, D.M., Morikawa, M., Zhao, C., Hirokawa, N., Lu, H., and Shen, K. (2016). Autoinhibition of a Neuronal Kinesin UNC-104/KIF1A Regulates the Size and Density of Synapses. Cell Rep 16, 2129-2141.

Niwa, S., Tao, L., Lu, S.Y., Liew, G.M., Feng, W., Nachury, M.V., and Shen, K. (2017). BORC Regulates the Axonal Transport of Synaptic Vesicle Precursors by Activating ARL-8. Curr Biol 27, 2569-2578 e2564.

Oka, M., Hashimoto, K., Yamaguchi, Y., Saitoh, S.I., Sugiura, Y., Motoi, Y., Honda, K., Kikko, Y., Ohata, S., Suematsu, M., et al. (2017). Arl8b is required for lysosomal degradation of maternal proteins in the visceral yolk sac endoderm of mouse embryos. J Cell Sci 130, 3568-3577.

Palomo-Guerrero, M., Fado, R., Casas, M., Perez-Montero, M., Baena, M., Helmer, P.O., Dominguez, J.L., Roig, A., Serra, D., Hayen, H., et al. (2019). Sensing of nutrients by CPT1C regulates late endosome/lysosome anterograde transport and axon growth. Elife 8.

Pu, J., Schindler, C., Jia, R., Jarnik, M., Backlund, P., and Bonifacino, J.S. (2015). BORC, a multisubunit complex that regulates lysosome positioning. Dev Cell 33, 176-188.

1101 Quintyne, N.J., Gill, S.R., Eckley, D.M., Crego, C.L., Compton, D.A., and Schroer, T.A. (1999).

1102 Dynactin is required for microtubule anchoring at centrosomes. J Cell Biol 147, 321-334.

1103 Raiborg, C., Wenzel, E.M., Pedersen, N.M., Olsvik, H., Schink, K.O., Schultz, S.W., Vietri, M., Nisi, V., Bucci, C., Brech, A., et al. (2015). Repeated ER-endosome contacts promote endosome translocation and neurite outgrowth. Nature 520, 234-238. 
1108 Rocha, N., Kuijl, C., van der Kant, R., Janssen, L., Houben, D., Janssen, H., Zwart, W., and Neefjes, J. (2009). Cholesterol sensor ORP1L contacts the ER protein VAP to control Rab7-RILP-p150 Glued and late endosome positioning. J Cell Biol 185, 1209-1225.

1111 Rosa-Ferreira, C., and Munro, S. (2011). Arl8 and SKIP act together to link lysosomes to kinesin1112 1. Dev Cell 21, 1171-1178.

1113 Rosa-Ferreira, C., Sweeney, S.T., and Munro, S. (2018). The small G protein Arl8 contributes to lysosomal function and long-range axonal transport in Drosophila. Biol Open 7.

1115 Roux, K.J., Kim, D.I., and Burke, B. (2013). BioID: a screen for protein-protein interactions. Curr Protoc Protein Sci 74, 1923 11-19 2314.

1117 Saric, A., Freeman, S.A., Williamson, C., Jarnik, M., Guardia, C.M., Fernandopulle, M.S., 1118 Gershlick, D.C., and Bonifacino, J.S. (2021). SNX19 restricts endolysosome motility 1119 through contacts with the endoplasmic reticulum. Nat Commun, in press.

1120 Schiefermeier, N., Scheffler, J.M., de Araujo, M.E., Stasyk, T., Yordanov, T., Ebner, H.L.,

Schneider, C.A., Rasband, W.S., and Eliceiri, K.W. (2012). NIH Image to ImageJ: 25 years of image analysis. Nat Methods 9, 671-675.

1126 Schroeder, C.M., Ostrem, J.M., Hertz, N.T., and Vale, R.D. (2014). A Ras-like domain in the light 1127 intermediate chain bridges the dynein motor to a cargo-binding region. Elife 3, e03351.

1128 Sindhwani, A., Arya, S.B., Kaur, H., Jagga, D., Tuli, A., and Sharma, M. (2017). Salmonella exploits the host endolysosomal tethering factor HOPS complex to promote its intravacuolar replication. PLoS Pathog 13, e1006700.

1131 Steffan, J.J., Dykes, S.S., Coleman, D.T., Adams, L.K., Rogers, D., Carroll, J.L., Williams, B.J., and progression. PLoS One 9, e87882.

1134 Sztul, E., Chen, P.W., Casanova, J.E., Cherfils, J., Dacks, J.B., Lambright, D.G., Lee, F.S., 1135 Randazzo, P.A., Santy, L.C., Schurmann, A., et al. (2019). ARF GTPases and their GEFs 1136 and GAPs: concepts and challenges. Mol Biol Cell 30, 1249-1271. 
1137 Terawaki, S., Camosseto, V., Prete, F., Wenger, T., Papadopoulos, A., Rondeau, C., Combes, A., Rodriguez Rodrigues, C., Vu Manh, T.P., Fallet, M., et al. (2015). RUN and FYVE

Tsuruta, F., and Dolmetsch, R.E. (2015). PIKfyve mediates the motility of late endosomes and domain-containing protein 4 enhances autophagy and lysosome tethering in response to Interleukin-4. J Cell Biol 210, 1133-1152.

Tuli, A., Thiery, J., James, A.M., Michelet, X., Sharma, M., Garg, S., Sanborn, K.B., Orange, J.S., Lieberman, J., and Brenner, M.B. (2013). Arf-like GTPase Arl8b regulates lytic granule polarization and natural killer cell-mediated cytotoxicity. Mol Biol Cell 24, 3721-3735.

Vilela, F., Velours, C., Chenon, M., Aumont-Nicaise, M., Campanacci, V., Thureau, A., Pylypenko, O., Andreani, J., Llinas, P., and Menetrey, J. (2019). Structural characterization of the RH1-LZI tandem of JIP3/4 highlights RH1 domains as a cytoskeletal motor-binding motif. Sci Rep 9, 16036.

Vukmirica, J., Monzo, P., Le Marchand-Brustel, Y., and Cormont, M. (2006). The Rab4A effector protein Rabip4 is involved in migration of NIH 3T3 fibroblasts. J Biol Chem 281, 3636036368.

Vukoja, A., Rey, U., Petzoldt, A.G., Ott, C., Vollweiter, D., Quentin, C., Puchkov, D., Reynolds,

Wang, G., Zhang, Q., Song, Y., Wang, X., Guo, Q., Zhang, J., Li, J., Han, Y., Miao, Z., and Li, F. (2015). PAK1 regulates RUFY3-mediated gastric cancer cell migration and invasion. Cell Death Dis 6, e1682.

Wei, Z., Sun, M., Liu, X., Zhang, J., and Jin, Y. (2014). Rufy3, a protein specifically expressed in 1162 Willett, R., Martina, J.A., Zewe, J.P., Wills, R., Hammond, G.R.V., and Puertollano, R. (2017). 1163 TFEB regulates lysosomal positioning by modulating TMEM55B expression and JIP4 recruitment to lysosomes. Nat Commun $8,1580$.

1165 Xie, R., Wang, J., Tang, W., Li, Y., Peng, Y., Zhang, H., Liu, G., Huang, X., Zhao, J., Li, A., et al. 1166 (2017). Rufy3 promotes metastasis through epithelial-mesenchymal transition in 1167 colorectal cancer. Cancer Lett 390, 30-38. 
1168 Zhu, H., Dai, W., Li, J., Xiang, L., Wu, X., Tang, W., Chen, Y., Yang, Q., Liu, M., Xiao, Y., et al. 1169 (2019). HOXD9 promotes the growth, invasion and metastasis of gastric cancer cells by 1170 transcriptional activation of RUFY3. J Exp Clin Cancer Res 38, 412.

1171 
Fig. 1

a
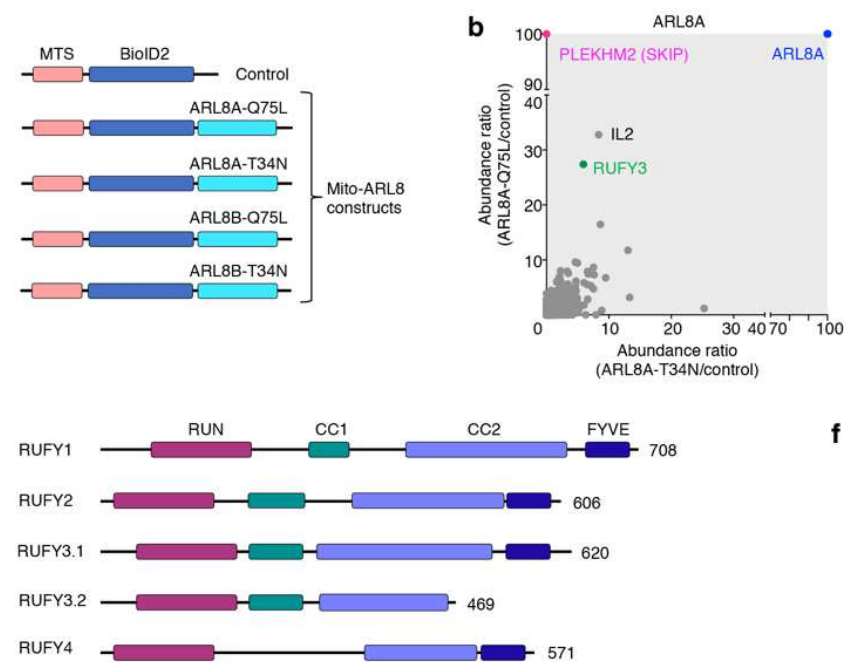

f
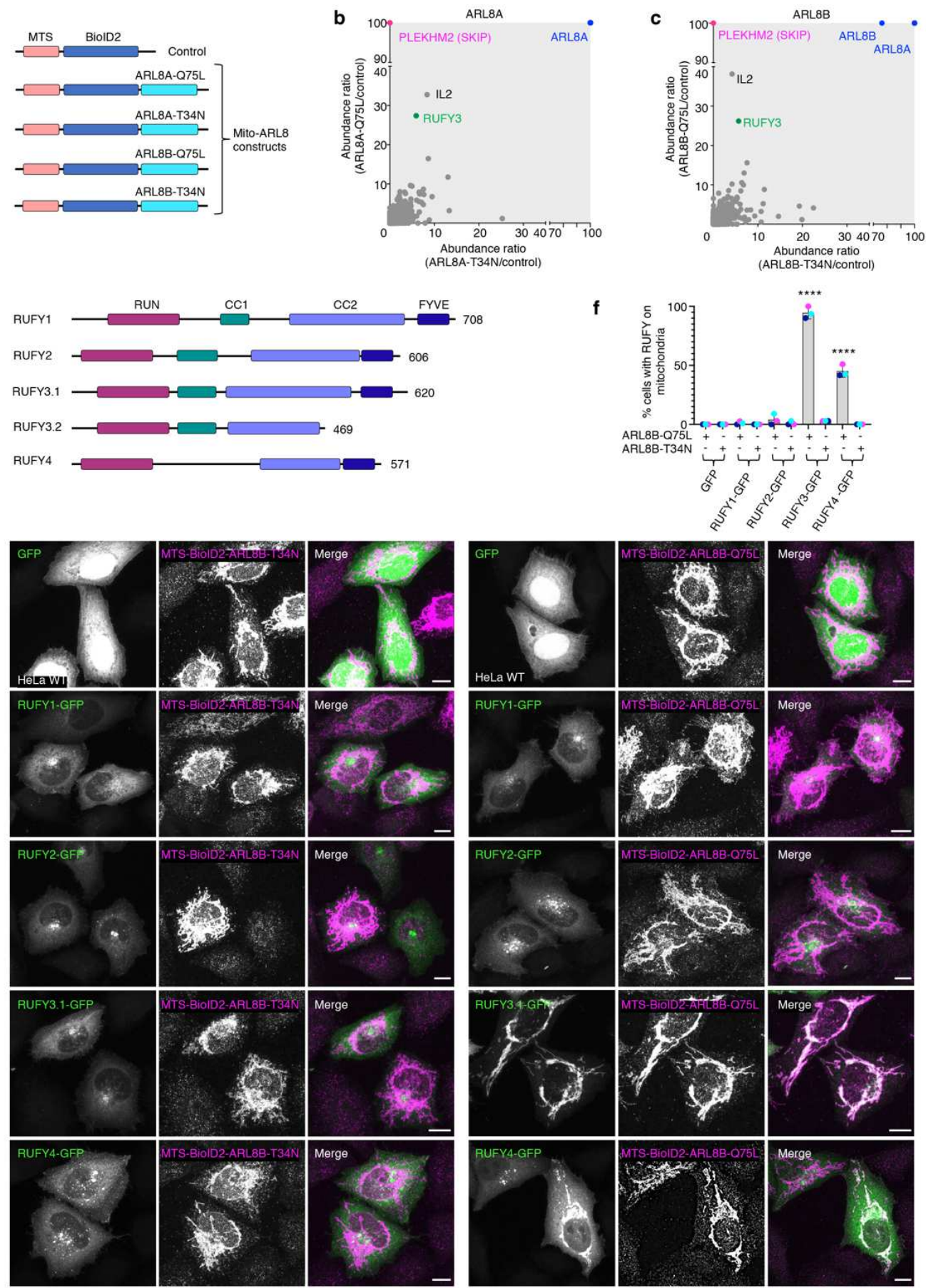

Merge
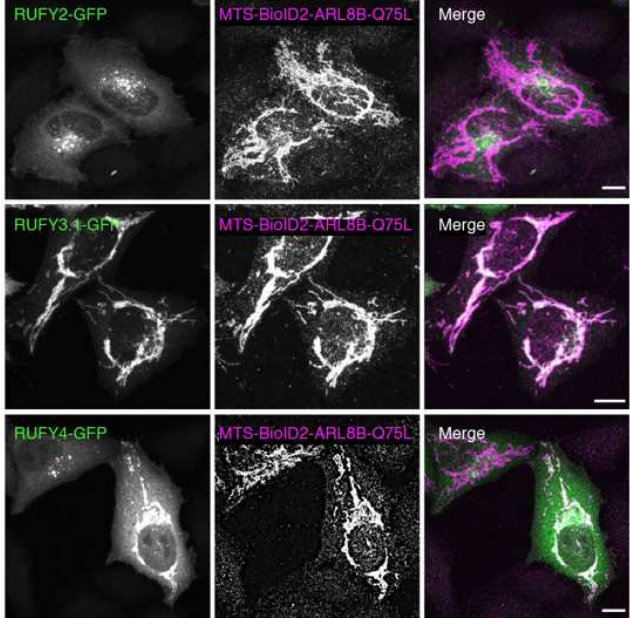

1172

1173 Fig. 1: Identification of RUFY3 and RUFY4 as ARL8 effectors. 
1174 a Schematic representation of control and Mito-ARL8 constructs used in MitoID. MTS:

1175 mitochondrial-targeting sequence from TOM20 (Kanaji et al., 2000); BioID2: humanized $A$.

1176 aeolicus biotin ligase (Kim et al., 2016). Constructs were expressed in HEK293T cells. b Graph

1177 showing the abundance of mass spectrometry hits identified for MTS-BioID2-ARL8A-

1178 Q75L/MTS-BioID2 control vs. MTS-BioID2-ARL8A-T34N/MTS-BioID2 control using MitoID. c

1179 Same as b for MTS-BioID2-ARL8B-Q75L / MTS-BioID2 control vs. MTS-BioID2-ARL8B-

1180 T34N/MTS-BioID2 control. Hits of interest in panels b and c are highlighted in color. $\mathbf{d}$ Domain

1181 organization of RUFY proteins in N- to C-terminal direction. RUN: RPIP8, UNC-14 and NESCA

1182 domain, CC1: coiled-coil 1 domain, CC2: coiled-coil 2 domain, FYVE: Fab1, YOTB, Vac1 and

1183 EEA1 domain. Amino-acid numbers are indicated. RUFY3.1 and RUFY3.2 are two spliceforms

1184 of RUFY3. e Immunofluorescence microscopy of HeLa cells co-expressing GFP or RUFY-GFP

1185 fusion proteins (green) together with MTS-BioID2-ALR8B-Q75L or -T34N. Fixed cells were

1186 stained with antibody to BioID2 (magenta), and imaged by confocal microscopy. Single

1187 channels are shown in grayscale. Scale bars: $10 \mu \mathrm{m}$. e Quantification of the percentage of cells in

1188 which RUFY proteins were re-localized to mitochondria from experiments such as that in panel

1189 e ( $n=3$ independent experiments; minimum of 300 cells per condition). Statistical significance

1190 was calculated using one-way ANOVA with multiple comparisons to the GFP control using

1191 Dunnett's test, ${ }^{* * *} \mathrm{p}<0.0001$. See also Supplementary Fig. 1 and Supplementary Dataset 1. 
Fig. 2

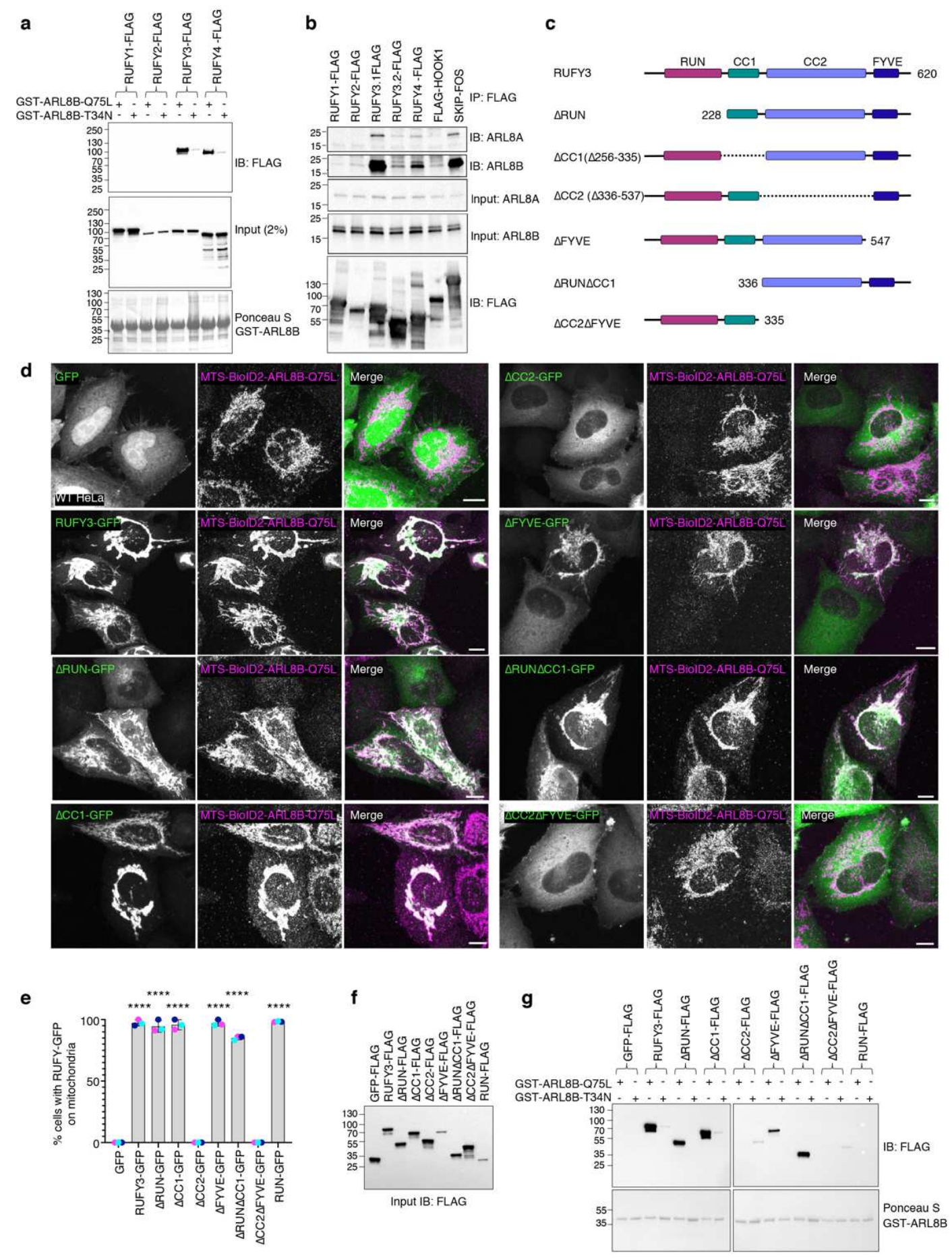

1193 Fig. 2: Biochemical evidence for the binding of RUFY3 and RUFY4 to ARL8 and dissection of 1194 RUFY3 domains required for ARL8 binding. 
1195 a Recombinant GST-ARL8B-Q75L and -T34N were purified using the GST tag and used to pull 1196 down the indicated RUFY-FLAG proteins expressed by transfection in HEK293T cells. GST

1197 proteins were detected by Ponceau S staining. IB: immunoblotting. b Extracts of HEK293T cells 1198 transfected with plasmids encoding the indicated FLAG- or FOS-tagged proteins were

1199 immunoprecipitated (IP) with anti-FLAG, and immunoblotted (IB) for endogenous ARL8A and 1200 ARL8B and the FLAG tag. In both panel $a$ and $b$, the positions of molecular mass markers (in $1201 \mathrm{kDa}$ ) are indicated at left. Both experiments are representative of two experiments with similar 1202 results. c Schematic representation of RUFY3 deletion constructs. Domain organization is as 1203 depicted in Fig. 1b. Amino-acid numbers are indicated. $\Delta$ stands for deletion. $\mathbf{d}$

1204 Immunofluorescence microscopy of HeLa cells expressing GFP or the RUFY3-GFP deletion 1205 constructs shown in panel c (green) together with MTS-BioID2-ALR8B-Q75L. Fixed cells were 1206 stained with antibody to BioID2 (magenta). Single channels are shown in grayscale. Scale bars:

$120710 \mu \mathrm{m}$. e Quantification of the percentage of cells in which RUFY-GFP proteins were re-localized 1208 to mitochondria ( $\mathrm{n}=3$ independent experiments; minimum of 300 cells per condition) from 1209 experiments such as that shown in panel d. Statistical significance compared to cells expressing 1210 GFP was calculated using one-way ANOVA with multiple comparisons with Dunnett's test. $1211^{* * * *} \mathrm{p}<0.0001$. f,g Recombinant GST-ARL8B-Q75L and -T34N were purified using the GST tag 1212 and used to pull down RUFY3-FLAG deletion constructs expressed by transfection in HEK293T 1213 cells. FLAG-tagged proteins in the input (f) and pull downs (g) were detected by 1214 immunoblotting (IB) for the FLAG epitope. Input GST-ARL8B-Q75L and -T34N were detected 1215 by Ponceau-S staining. The positions of molecular mass markers (in $\mathrm{kDa}$ ) in panels $\mathrm{f}$ and $\mathrm{g}$ are 1216 indicated at left. This experiment is representative of two experiments with similar results. 
Fig. 3

a
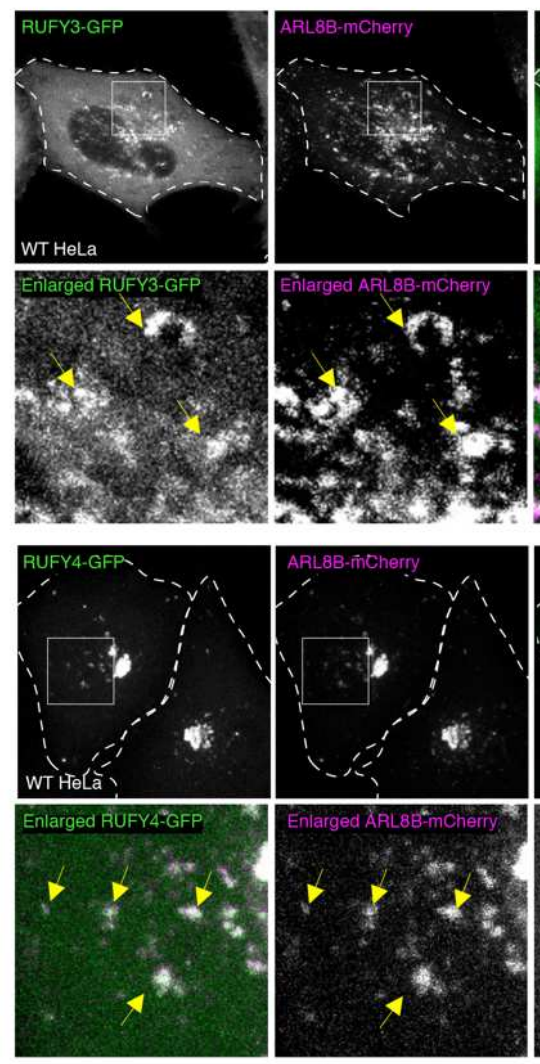

b

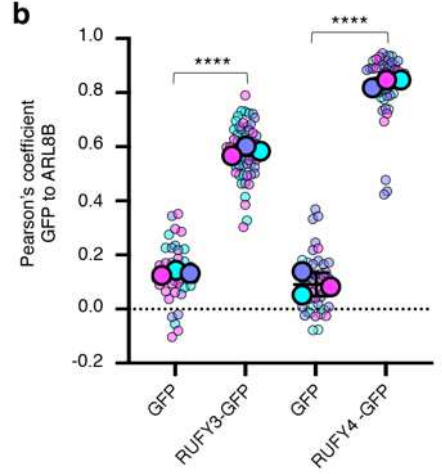

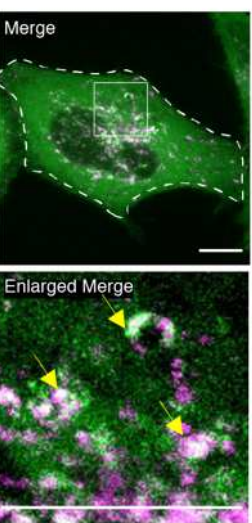

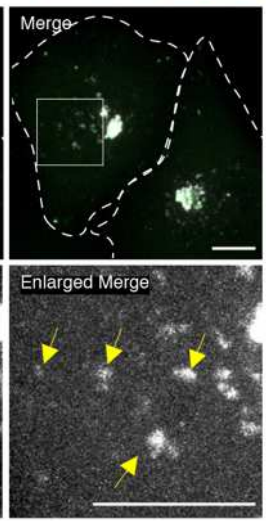

d

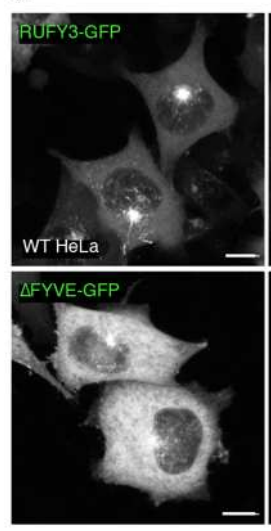

c
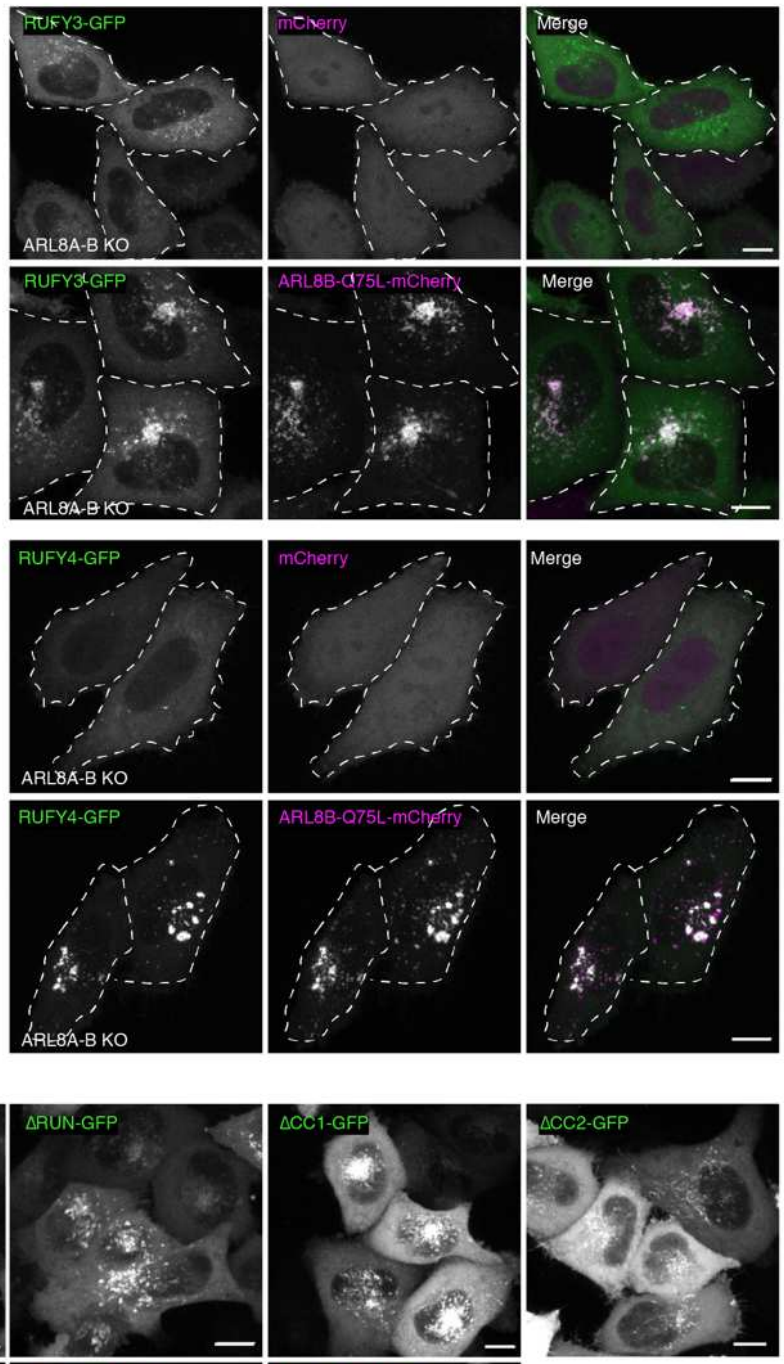

$\triangle R U N \triangle C C 1-G F P$

$\triangle C C 2 \triangle F Y V E-C F P$
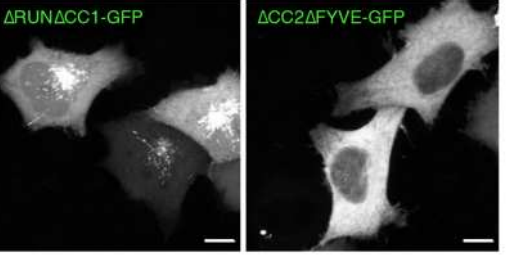

1218 Fig. 3: ARL8 recruits RUFY3 and RUFY4 to a cluster of juxtanuclear vesicles.

1219 a Live-cell imaging of HeLa cells co-expressing RUFY3-GFP or RUFY4-GFP (green) with ARLB-

1220 mCherry (magenta). Cell edges are outlined by dashed lines. Scale bars: $10 \mu \mathrm{m}$. The lower rows

1221 are 4.7-fold (RUFY3) and 3.7-fold (RUFY4) enlargements of the boxed areas. Arrows indicate

1222 vesicles where RUFY-GFP proteins and ARLB-mCherry co-localize. Single channels are shown 
1223 in grayscale. $\mathbf{b}$ Co-localization of GFP (control), RUFY3-GFP or RUFY4-GFP with ARL8B1224 mCherry from experiments such as that in panel a. The graph shows the mean $\pm \mathrm{SD}$ and the 1225 individual data points from 3 independent experiments. Statistical significance was calculated 1226 using Student's t-test. ${ }^{* * * *} \mathrm{p}<0.0001$. c ARL8A-B-KO cells were co-transfected with plasmids 1227 encoding RUFY3-GFP or RUFY4-GFP (green) with mCherry (control) or ARL8B-Q75L-mCherry 1228 (magenta). Cells were imaged live by confocal microscopy. Single channels are shown in 1229 grayscale. Scale bars: $10 \mu \mathrm{m}$. $\mathbf{d}$ Live-cell imaging of HeLa cells expressing the RUFY3-GFP 1230 deletion constructs shown in Fig. 2c. Images are in grayscale. Scale bars: $10 \mu \mathrm{m}$. 
Fig. 4
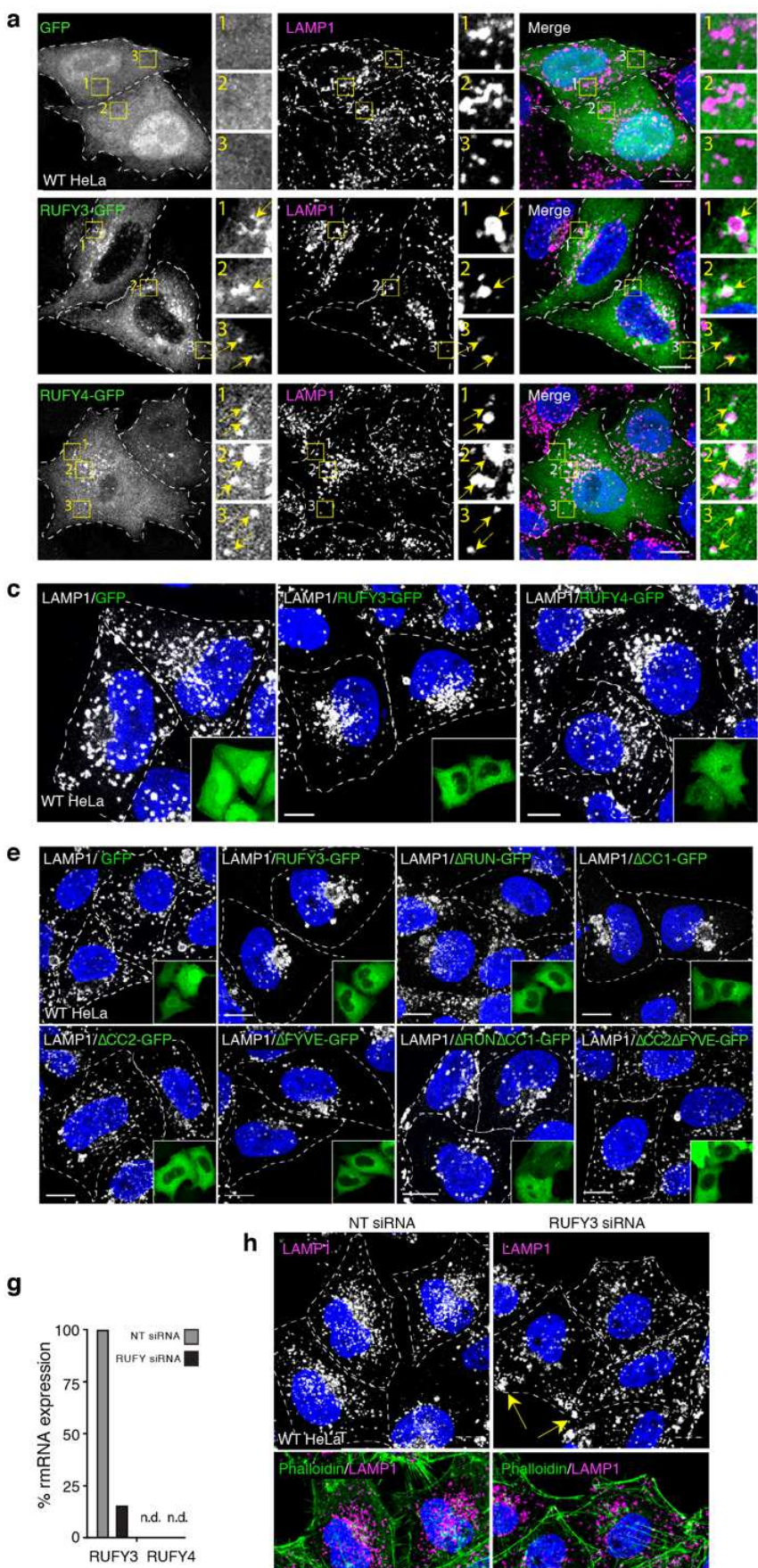

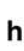

h

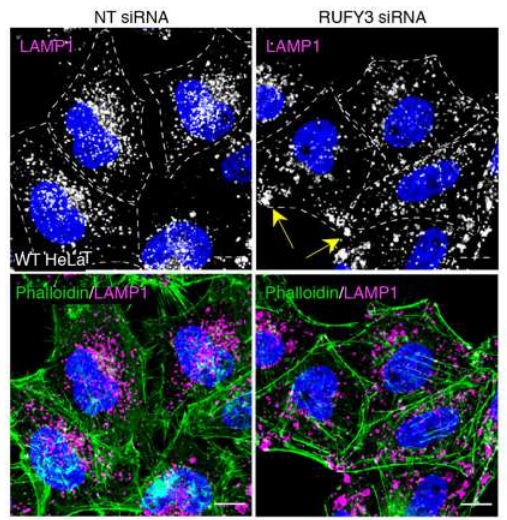

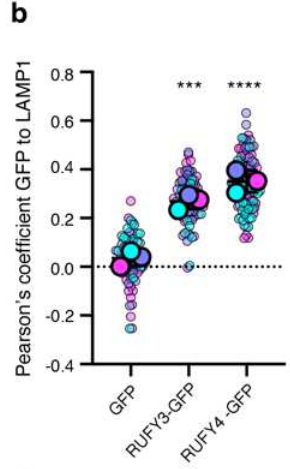

d
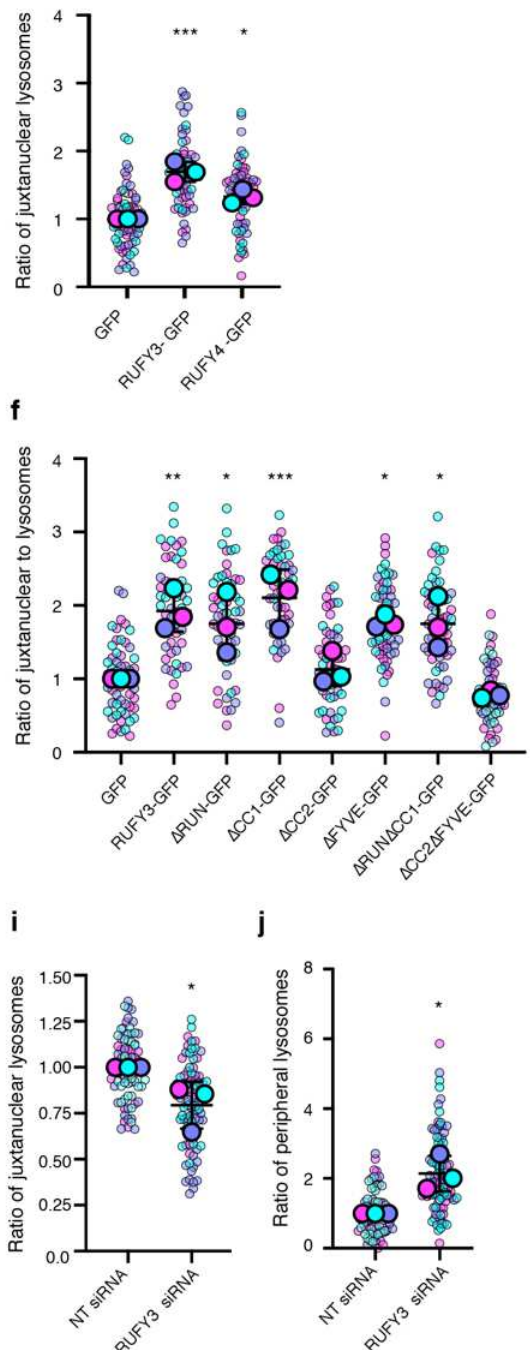

1233 Fig. 4: RUFY3 and RUFY4 promote juxtanuclear localization of lysosomes. 
1234 a Co-localization of RUFY3-GFP or RUFY4-GFP with endogenous LAMP1.

1235 Immunofluorescence microscopy of HeLa cells transfected with plasmids expressing GFP

1236 (control), RUFY3-GFP or RUFY4-GFP (green), fixed and immunostained for endogenous

1237 LAMP1 (magenta). Nuclei were stained with DAPI (blue). Cell edges are outlined by dashed

1238 lines. Scale bars: $10 \mu \mathrm{m}$. Insets show 3-fold enlargements of the boxed areas. Single channels are

1239 shown in grayscale. Arrows indicate vesicles where RUFY-GFP proteins co-localize with

1240 LAMP1. b Co-localization of GFP, RUFY3-GFP or RUFY4-GFP with endogenous LAMP1 from

1241 experiments such as that in a. The graph shows the mean \pm SD from and the individual data

1242 points from 3 independent experiments. Statistical significance was calculated using one-way

1243 ANOVA with multiple comparisons to the GFP control using Dunnett's test. ${ }^{* * *} \mathrm{p}<0.001$, ${ }^{* * * *}$

$1244 \mathrm{p}<0.0001$. c Overexpression of RUFY3-GFP or RUFY4-GFP causes juxtanuclear clustering of

1245 lysosomes. This experiment was done as described for panel a. Endogenous LAMP1 staining is

1246 shown in grayscale and GFP images in green (inset). Nuclei were stained with DAPI (blue). Cell

1247 edges are outlined by dashed lines. Scale bars: $10 \mu \mathrm{m}$. d, Quantification of the ratio of

1248 juxtanuclear LAMP1 to total LAMP1 calculated by shell analysis from experiments such as

1249 those in panels $a$ and $b$. The graph shows the mean \pm SD and the individual data points from 3

1250 independent experiments. Statistical significance was calculated using one-way ANOVA with

1251 multiple comparison to the GFP control using Dunnett's test. ${ }^{*} \mathrm{p}<0.05$, ${ }^{* * *} \mathrm{p}<0.001$. e

1252 Immunofluorescence microscopy of HeLa cells transfected with GFP (control) or RUFY3-GFP

1253 deletions constructs depicted in Fig. 2c (green in the insets), fixed and immunostained for

1254 endogenous LAMP1 (grayscale). Nuclei were stained with DAPI (blue). Cell edges are outlined

1255 by dashed lines. Scale bars: $10 \mu \mathrm{m}$. f Quantification, as described for panel d, of the effect of

1256 RUFY3-GFP deletion constructs on the distribution of LAMP1 from 3 independent experiments

1257 such as that shown in panel d. Statistical significance was calculated using one-way ANOVA

1258 with multiple comparisons to GFP using Dunnett's test. ${ }^{*} \mathrm{p}<0.05,{ }^{* *} \mathrm{p}<0.01,{ }^{* * *} \mathrm{p}<0.001$. g qRT-

1259 PCR of mRNA expression of RUFY3 and RUFY4 relative to actin in HeLa cells treated with non-

1260 targeting (NT) or RUFY3/4 siRNAs. n.d., not detected. $\mathbf{h}$ Immunofluorescence microscopy of

1261 HeLa cells treated with non-targeting (NT) or RUFY3 siRNA and stained with antibodies to

1262 endogenous LAMP1 (grayscale and magenta) and Alexa fluor 546-conjugated phalloidin

1263 (green) to highlight cell edges. Nuclei were stained with DAPI (blue). Cell edges are outlined by

1264 dashed lines. Scale bars: $10 \mu \mathrm{m}$. i Quantification, as described for panel d, of the effect of NT or

1265 RUFY3 siRNA on the juxtanuclear localization of LAMP1 from 3 independent experiments such

1266 as that shown in panel h. $\mathbf{j}$ Quantification of the ratio of peripheral LAMP1 to total LAMP1

1267 calculated by shell analysis from 3 independent experiments such as that shown in panel $\mathrm{h}$.

1268 
Fig. 5
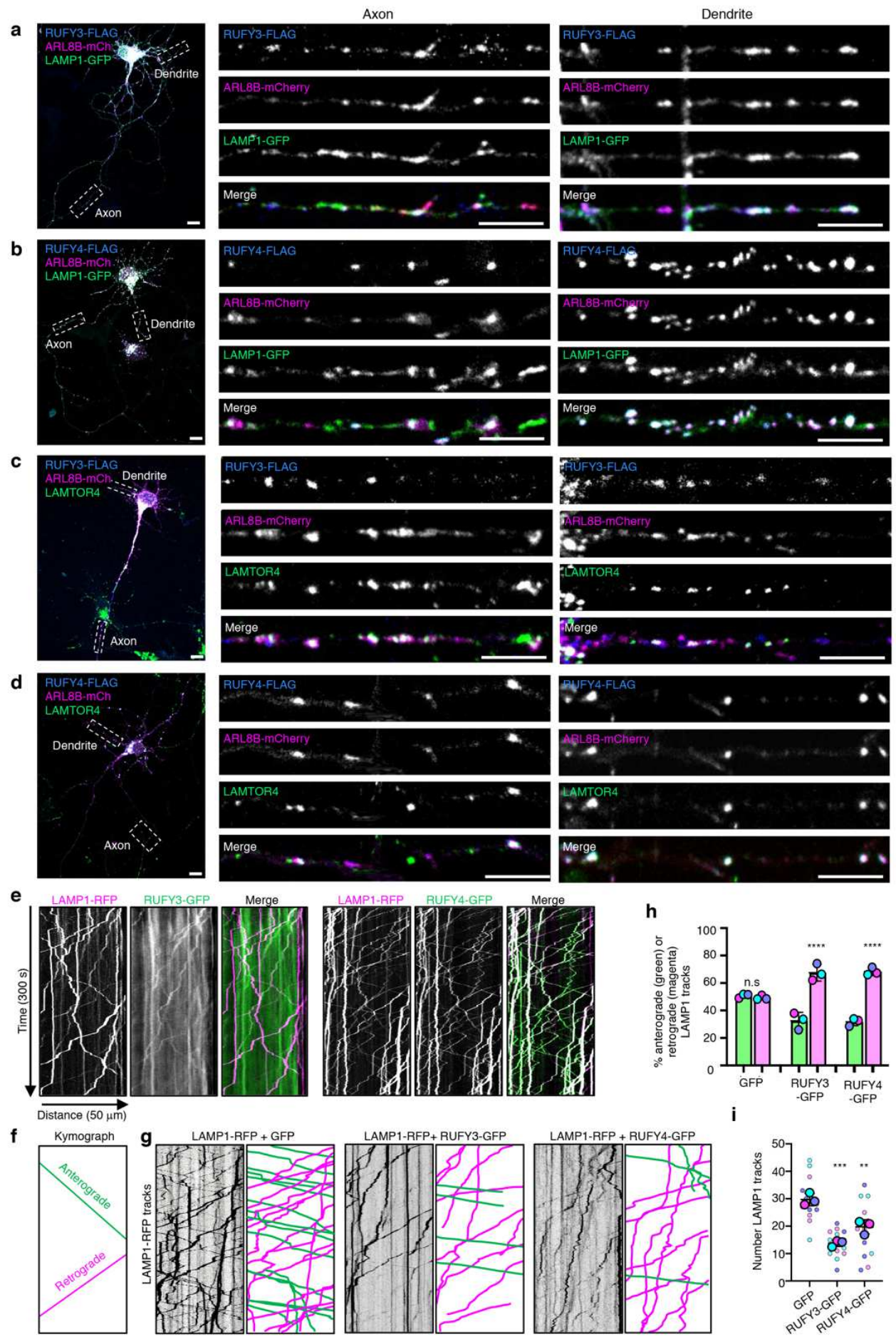

1270 Fig. 5: RUFY3 and RUFY4 promote axonal retrograde transport. 
$1271 \mathbf{a}, \mathbf{b}$ Immunofluorescence microscopy of rat hippocampal neurons transfected with plasmids 1272 encoding RUFY3-FLAG (a) or RUFY4-FLAG (b) along with ARL8B-mCherry and LAMP1-GFP.

1273 Neurons were fixed, permeabilized and RUFY-FLAG proteins were detected by

1274 immunostaining with antibody to the FLAG epitope (blue), and ARL8B-mCherry (magenta)

1275 and LAMP1-GFP (green) by their intrinsic fluorescence. Images on the left show neurons (Scale

1276 bars: $10 \mu \mathrm{m}$ ) with boxes indicating axons and dendrites that are enlarged on the right (Scale

1277 bars: $5 \mu \mathrm{m})$. c,d Same as panels a and b, but neurons were immunostained with antibody to

1278 endogenous LAMTOR4 (green) instead of imaged for LAMP1-GFP. e Rat hippocampal neurons

1279 were transfected with plasmids encoding LAMP1-RFP (magenta) along with RUFY3-GFP or

1280 RUFY4-GFP (green), axons were imaged live by spinning-disk confocal microscopy, and

1281 trajectories of fluorescent particles were represented as kymographs. Single channels are

1282 represented in grayscale. $\mathbf{f}$ Lines with negative or positive slopes in the kymographs correspond

1283 to vesicles moving in anterograde (green) or retrograde (magenta) directions, respectively. $\mathbf{g}$

1284 Kymographs representing the movement of LAMP1-RFP (grayscale) in live cells co-expressing

1285 GFP, RUFY3-GFP or RUFY4-GFP (not shown), with manual analysis of the tracks according to

1286 the convention in panel f. h Quantification of the percentage of anterograde (green) and

1287 retrograde (magenta) movement of LAMP1-RFP vesicles in neurons expressing GFP, RUFY3-

1288 GFP or RUFY4-GFP from experiments such as that in panel g. Values are the mean \pm SD from 3

1289 independent experiments with a total of 15 neurons analyzed per condition and counting a total

1290 of 445 (GFP), 206 (RUFY3-GFP), 282 (RUFY4-GFP) LAMP1-RFP motile events per condition.

1291 Statistical significance was calculated using one-way ANOVA with multiple comparisons using

1292 Tukey's test. ${ }^{* * *} \mathrm{p}<0.0001$; n.s., not significant. i Quantification of the total number of LAMP1-

1293 RFP tracks in neurons expressing GFP, RUFY3-GFP or RUFY4-GFP from experiments such as

1294 that in panel $g$. The graph shows the mean \pm SD and the individual data points from 3

1295 independent experiments. Statistical significance was calculated using one-way ANOVA with

1296 multiple comparisons to the GFP control using Dunnett's test. ${ }^{* *} \mathrm{p}<0.001,{ }^{* *} \mathrm{p}<0.01$. See also

1297 Supplementary Movie 1. 
Fig. 6
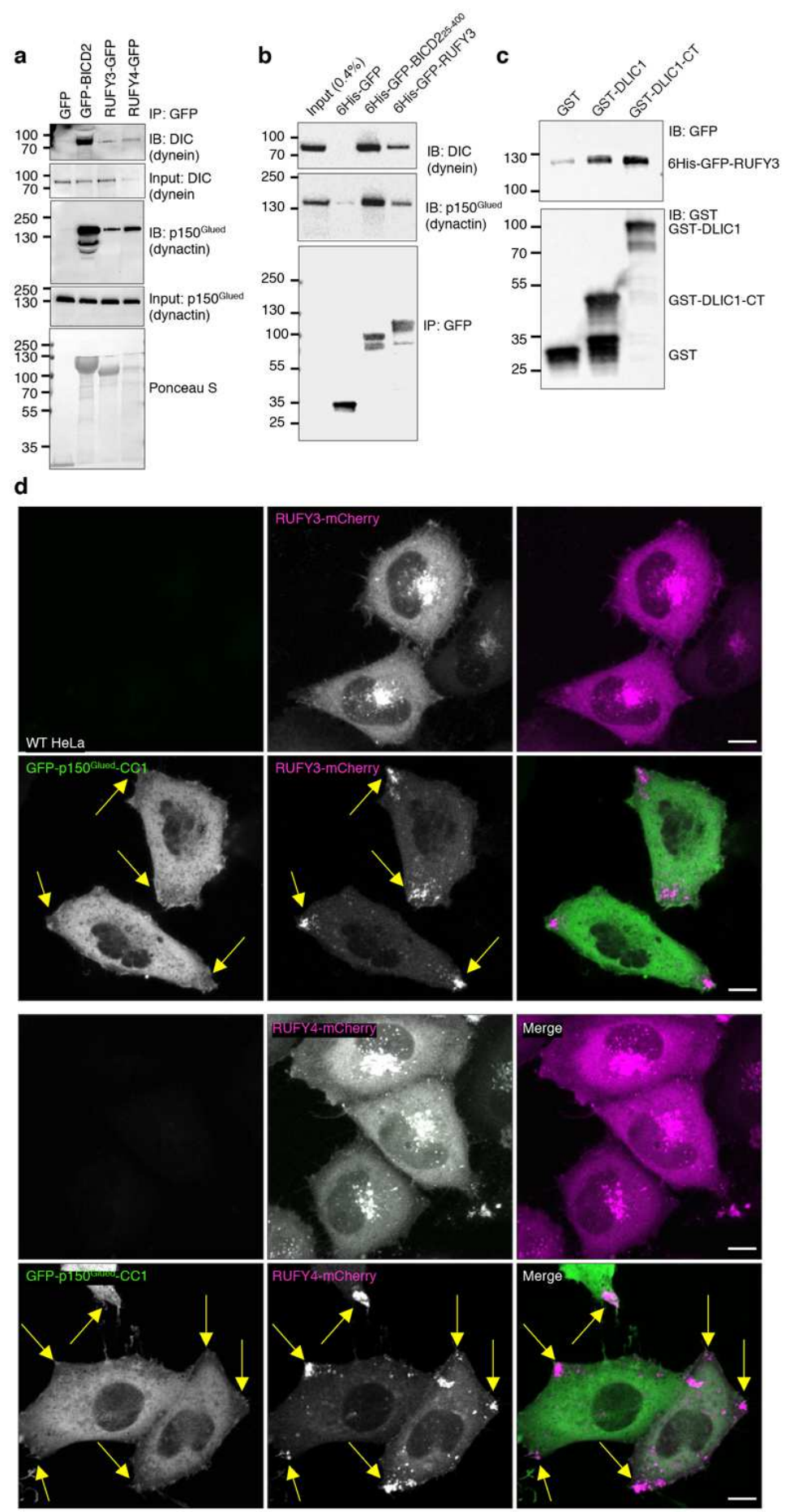

1299 Fig. 6: RUFY3 and RUFY4 bind dynein-dynactin.

1300 a HEK293T cells were transfected with plasmids encoding GFP (negative control), GFP-BICD2 1301 (positive control), RUFY3-GFP or RUFY4-GFP. Cell extracts were analyzed by 
1302 immunoprecipitation (IP) with antibody to GFP followed by immunoblotting for endogenous

1303 dynein intermediate chain (DIC) and the endogenous p150 Glued subunit of dynactin. Ponceau S

1304 staining shows the levels of immunoprecipitated GFP-tagged proteins. The experiment shown

1305 in this panel is one of two with similar results. $\mathbf{b}$ Extracts of HEK293T cells were incubated with

1306 recombinant 6His-Strep-GFP (negative control), His6-Strep-GFP-BICD2 $25-400$ (positive control) or

1307 6His-Strep-GFP-RUFY3, pulled down with Strep-Tactin agarose, and immunoblotted for

1308 endogenous dynein intermediate chain (DIC), the endogenous p150 Glued of dynactin, or GFP.

1309 The GFP used to make these constructs is a variant named sfGFP, for super-folder GFP. The

1310 experiment shown in this panel is one of two with similar results. c Glutathione-Sepharose

1311 preloaded with purified, recombinant GST (negative control), GST-DLIC1 or GST-DLIC1-CT (C-

1312 terminal domain) were incubated with purified, recombinant 6His-sfGFP-RUFY3. Bound

1313 proteins were detected by immunoblotting with antibodies to GFP and GST. The positions of

1314 molecular mass markers (in $\mathrm{kDa}$ ) in panels a-c are indicated at left. $\mathbf{d}$ Live-cell imaging of HeLa

1315 cells co-expressing RUFY3-mCherry or RUFY4-mCherry (magenta) without or with GFP-

1316 p150 Glued_CC1 (green). Single channel images are shown in grayscale. Scale bars: $10 \mu \mathrm{m}$. Arrows

1317 point to RUFY proteins at cell tips. This experiment is one of two with similar results. 
Fig. 7
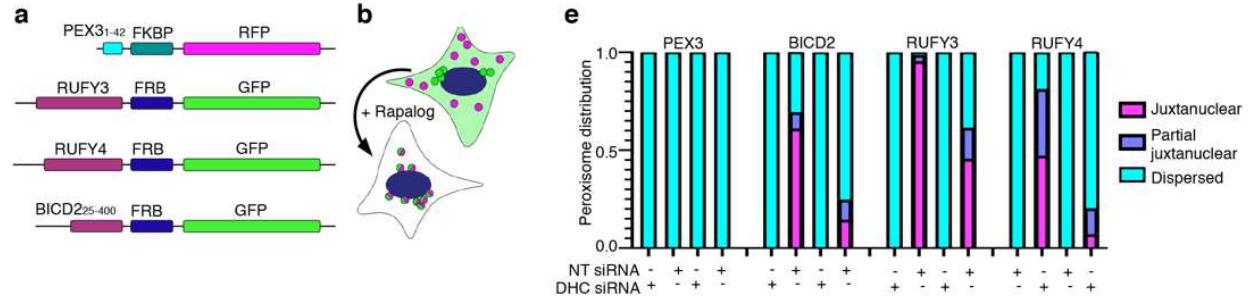

$$
\text { C }
$$

Non-targeting (NT) siRNA

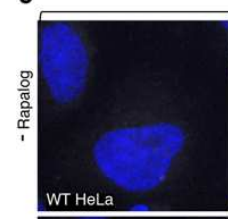

PPEX3:-42-FKBP-RFP

웛
$\frac{0}{\pi}$
$\frac{0}{\pi}$
ㄸ
+
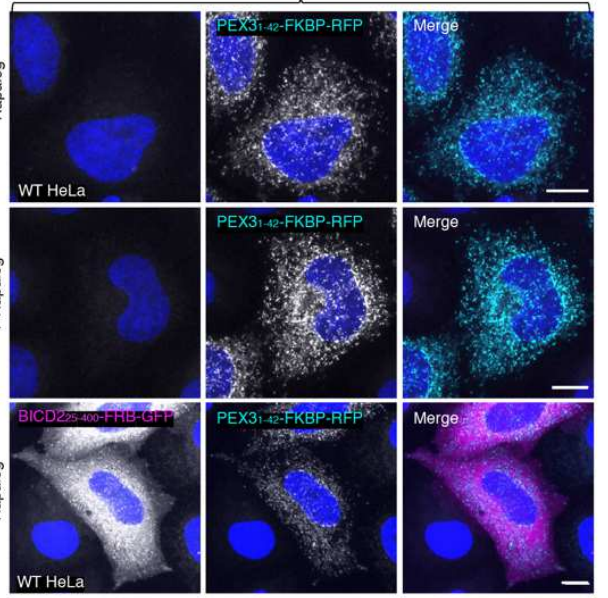

Merge
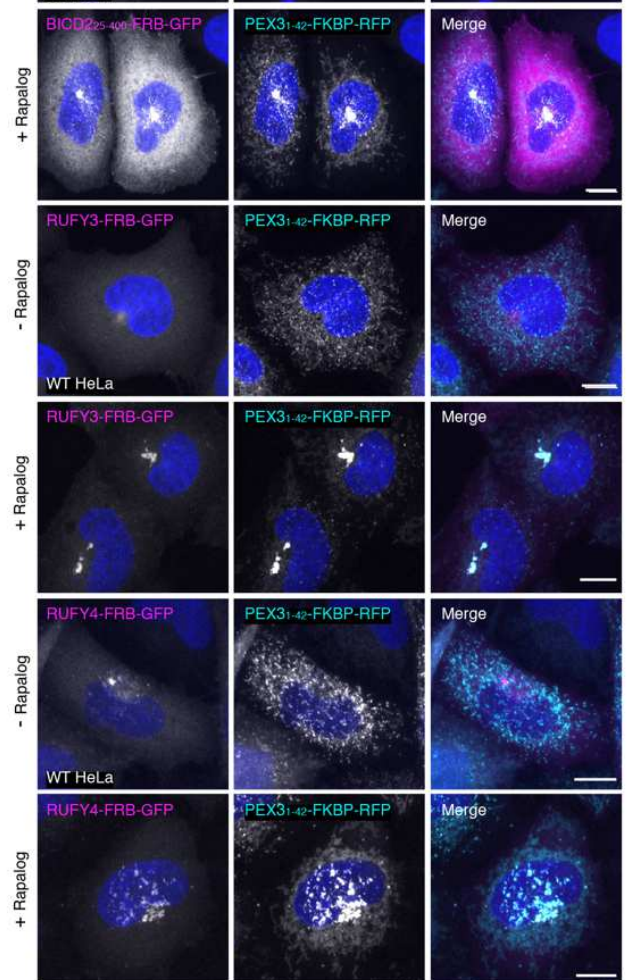

d Dynein Heavy Chain (DHC) siRNA
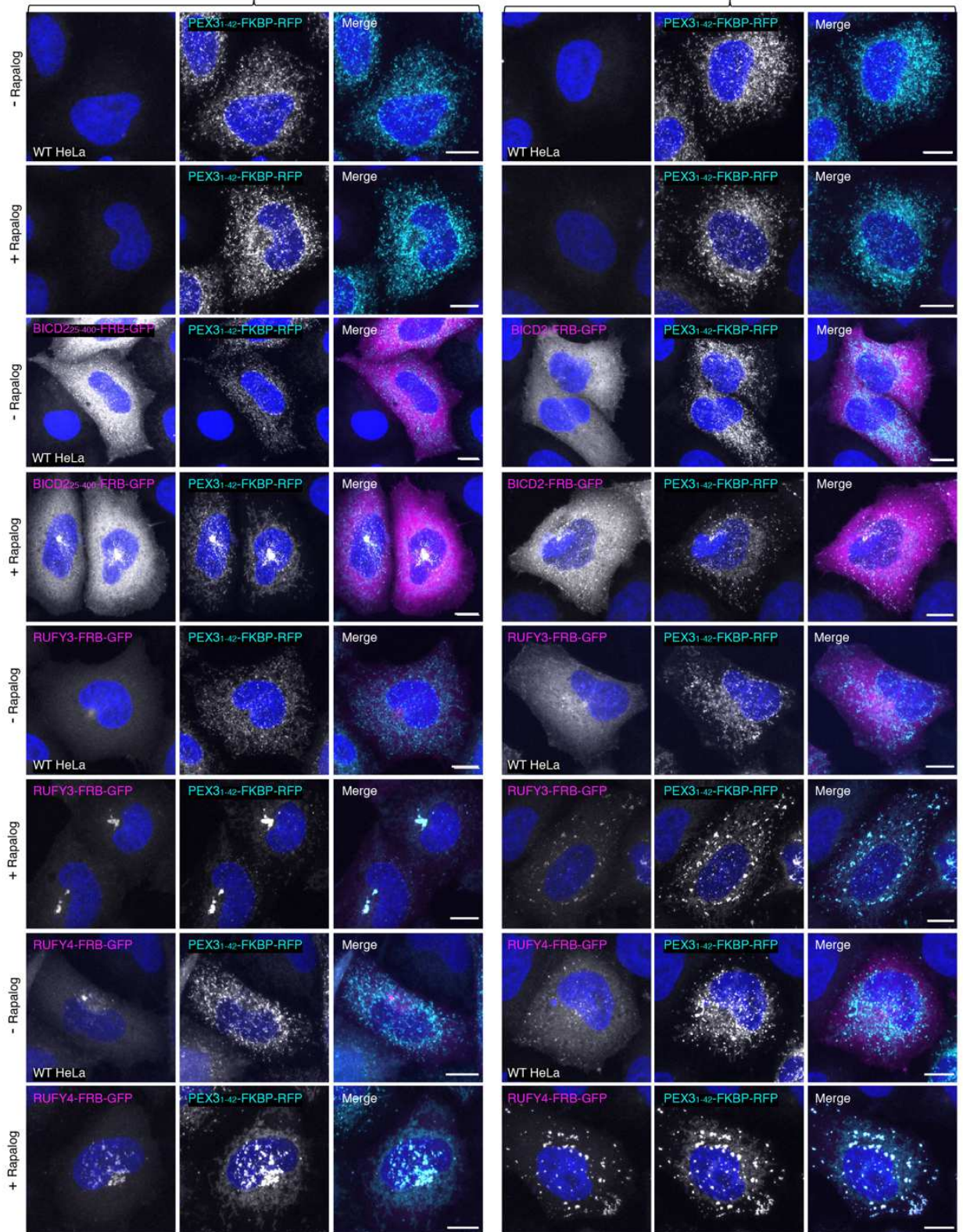

1319 Fig. 7: Targeting of RUFY3 and RUFY4 to peroxisomes promotes their juxtanuclear 1320 accumulation in a dynein-dependent manner. 
1321 a Schematic representation of constructs used in the peroxisome re-localization assay. PEX3-42: 1322 peroxisomal-targeting signal from PEX3; FKBP: FK506-binding protein; FRB: FKBP rapamycin 1323 binding. Constructs are represented in the N- to C-terminal direction. BICD2 $25-400$ was used as a 1324 positive control for a dynein-dynactin adaptor. FKBP binds to FRB upon addition of rapalog. $\mathbf{b}$ 1325 Schematic representation of the rapalog-induced juxtanuclear re-localization of peroxisomes 1326 labeled by PEX3 1-42 $_{2}$-FKBP-RFP (magenta) by a hypothetical dynein-dynactin adaptor fused to 1327 FRB and GFP (green). c,d Fluorescence microscopy of HeLa cells treated with non-targeting 1328 (NT) siRNA (c) or dynein heavy chain (DHC) siRNA (d), co-transfected with plasmids encoding 1329 the indicated proteins, and incubated for $1 \mathrm{~h}$ without (-) or with (+) $0.5 \mu \mathrm{M}$ rapalog. Nuclei were 1330 stained with DAPI. Scale bars: $10 \mu \mathrm{m}$. This experiment is representative of 3 experiments with 1331 similar results. $\mathrm{e}$ Cells from experiments such as that shown in panels $\mathrm{c}$ and $\mathrm{d}$ (a minimum of 1332200 cells from 2-3 independent experiments) were visually scored for the distribution of 1333 peroxisomes. 
Fig. 8
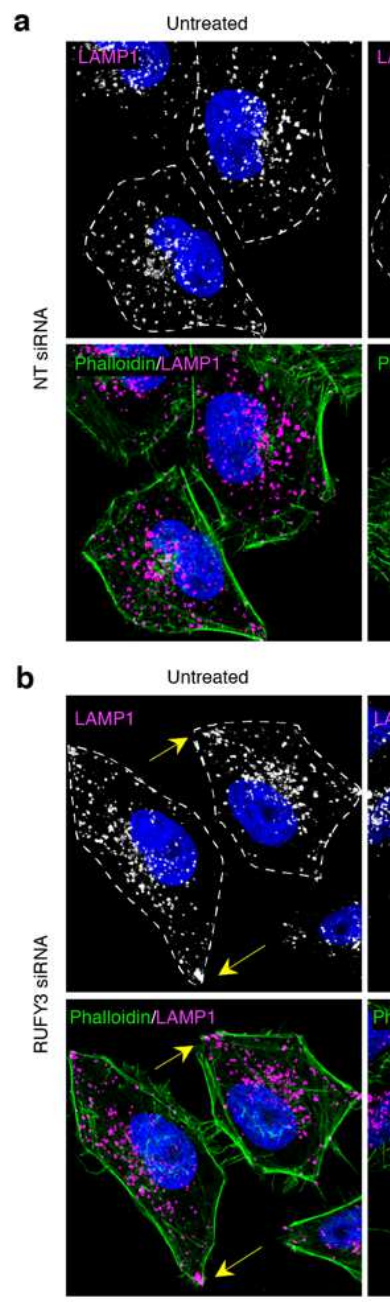
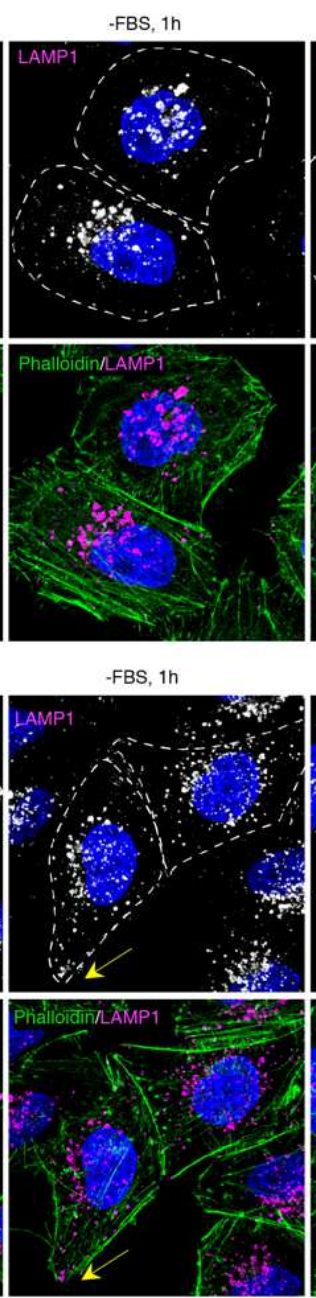

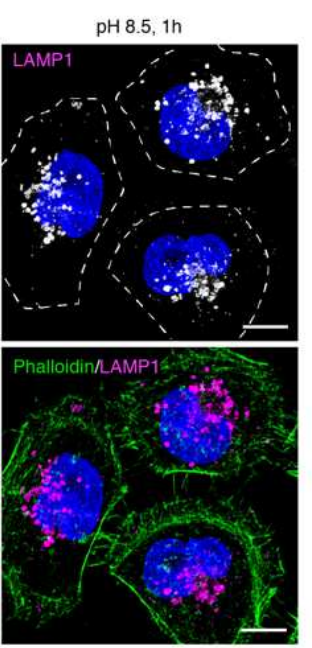

$\mathrm{pH} 8.5,1 \mathrm{~h}$

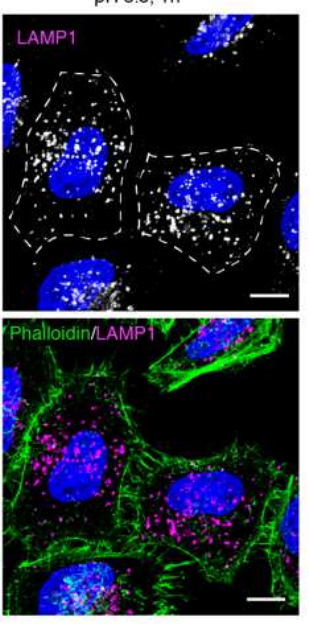

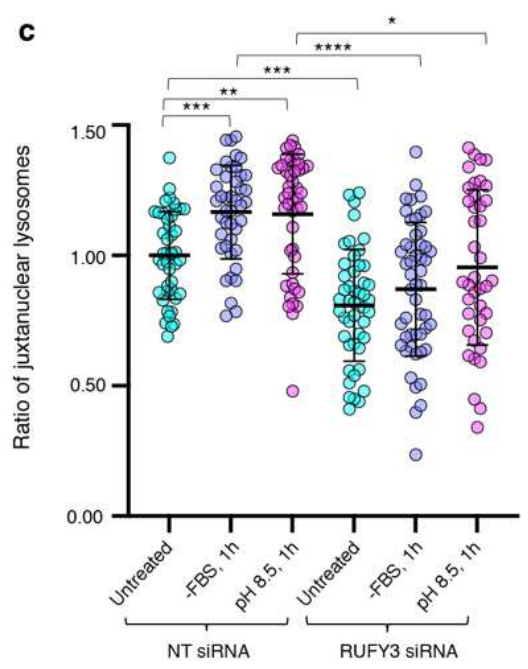

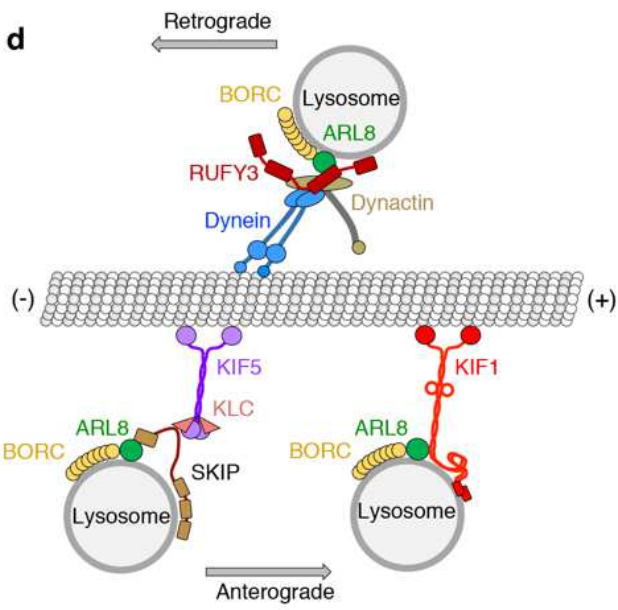

1335 Fig. 8: RUFY3 is required for juxtanuclear clustering of lysosomes induced by serum 1336 starvation cytoplasmic alkalinization.

$1337 \mathbf{a}, \mathbf{b}$ HeLa cells were treated with non-targeting (a) or RUFY3 siRNA (b) for $96 \mathrm{~h}$, and incubated 1338 for $1 \mathrm{~h}$ at $37^{\circ} \mathrm{C}$ in serum-free DMEM or for $1 \mathrm{~h}$ in regular culture medium adjusted to $\mathrm{pH} 8.5$, as 1339 indicated in the figure. Cells were then fixed, permeabilized and immunostained with antibody

1340 to endogenous LAMP1 (grayscale and magenta) and Alexa Fluor 546-conjugated phalloidin

1341 (green). Nuclei were stained with DAPI (blue). Arrows indicate accumulation of lysosomes at

1342 cell tips caused by RUFY3 depletion. Scale bars: $10 \mu \mathrm{m}$. c Quantification of the ratio of

1343 juxtanuclear LAMP1 to total LAMP1 calculated by shell analysis from the experiment shown in 
1344 panels a and $b$, normalized to untreated cells in regular culture medium. The graph shows the 1345 mean \pm SD and the individual values from 40-50 cells analyzed per condition. Statistical 1346 significance was calculated by Brown-Forsythe and Welch ANOVA tests with multiple 1347 comparisons using Dunnett's T3 test. ${ }^{* * *} \mathrm{p}<0.0001,{ }^{* * *} \mathrm{p}<0.001,{ }^{* *} \mathrm{p}<0.01,{ }^{* *} \mathrm{p}<0.05 . \mathbf{d}$

1348 Schematic representation of the role of ARL8 in regulating both retrograde or anterograde of 1349 lysosomes through interactions with different effectors. BORC promotes recruitment of ARL8 to 1350 lysosomes (Pu et al., 2015). In turn, ARL8 recruits RUFY3 (or RUFY4), which serves as an 1351 adaptor for dynein-dynactin, thus driving transport from the plus to the minus end of 1352 microtubules (i.e., retrograde transport) (this study). Alternatively, ARL8 recruits kinesin-1 1353 (KIF5 $\left.2-\mathrm{KLC}_{2}\right)$ via SKIP or kinesin-3 (KIF1) directly, driving transport from the minus to the plus 1354 end of microtubules (Farias et al., 2017; Guardia et al., 2016; Pu et al., 2015; Rosa-Ferreira and 1355 Munro, 2011). 


\section{Supplementary Fig. 1}

a

Transfection of HEK293T cells with 1 control and 4 mito-ARL8 constructs (Fig. 1a)

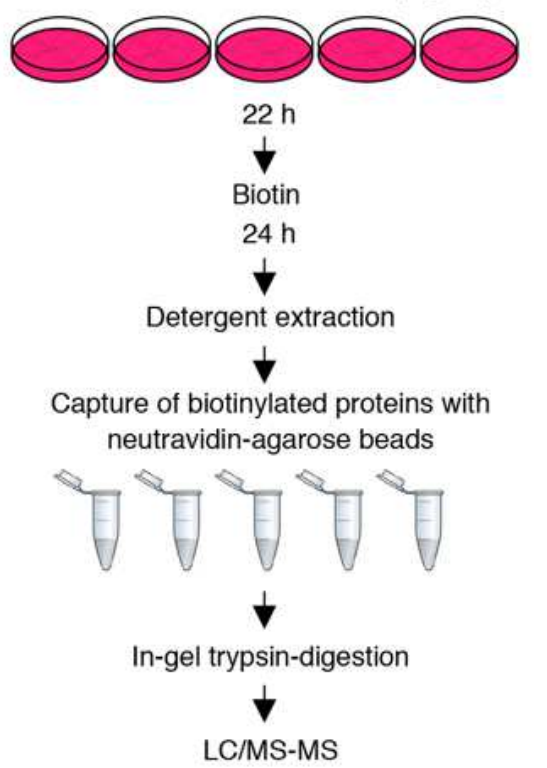

b

\begin{tabular}{|l|l|}
\hline RUFY3.1 peptides & Amino acids \\
\hline [R].ELDDISLTPDPEPTHEDPNYLMANER.[M] & $44-69$ \\
\hline [K].QDFGDKINSLQLEVEELTR.[Q] & $466-484$ \\
\hline [K].INSLQLEVEELTR.[Q] & $472-484$ \\
\hline [R].NQLELELK.[Q] & $487-494$ \\
\hline [R].LQPHPMDEQDQLLLSEKPQLCQLCQEDGSLTK.[N] & $542-573$ \\
\hline [K].NCSGTFCDACSTNELPLPSSIK.[L] & $578-599$ \\
\hline
\end{tabular}

C

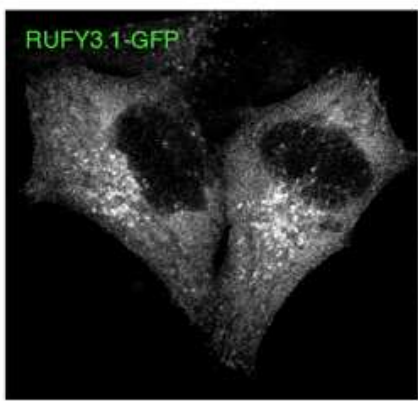

\section{RUFY3.2-GFP}

1358 Supplementary Fig. 1: MitoID procedure and identification of RUFY3.1.

1359 a Workflow of the MitoID procedure. $\mathbf{b}$ Peptides and amino-acid numbers specific for the

1360 RUFY3.1 spliceform identified by mass spectrometry. $\mathbf{b}$ Confocal microscopy of HeLa cells

1361 transfected with plasmids encoding RUFY3.1-GFP or RUFY3.2-GFP. Scale bars: $10 \mu \mathrm{m}$. Notice

1362 that RUFY3.1-GFP associates with vesicles, whereas RUFY3.2-GFP is cytosolic. 
1363 Supplementary Dataset 1: List of proteins identified in the MitoID mass spectrometry

1364 A-TN: Mito-BioID2-ARL8A-T34N, A-QL: Mito-BioID2-ARL8A-Q75L, B-TN: Mito-BioID2-

1365 ARL8B-T34N. B-QL: Mito-BioID2-ARL8B-Q75L. Ctrl: MitoID-BioID2 control.

1366

1367 Supplementary Movie M1: RUFY3 and RUFY4 co-move with LAMP1 labeled lysosomes in 1368 the axon.

1369 DIV5 rat hippocampal neurons co-expressing LAMP1-RFP with RUFY3-GFP or RUFY4-GFP

1370 were imaged using a spinning-disk confocal microscopy. Dual-color images of $50 \mu \mathrm{m}$ adjacent

1371 to the axon initial segment (AIS) at a speed of 15 frame per second for 300 seconds without any 1372 delay. Notice the co-movement of LAMP1-RFP with RUFY3-GFP or RUFY4-GFP. 


\section{Supplementary Files}

This is a list of supplementary files associated with this preprint. Click to download.

- SupplementaryDataset1.xlsx

- Supplementarymovie1.mov 